Probing background ionization: positive streamers with varying pulse repetition rate and with a radioactive admixture

This article has been downloaded from IOPscience. Please scroll down to see the full text article.

2011 J. Phys. D: Appl. Phys. 44455201

(http://iopscience.iop.org/0022-3727/44/45/455201)

View the table of contents for this issue, or go to the journal homepage for more

Download details:

IP Address: 192.16.184.152

The article was downloaded on 31/10/2011 at 14:57

Please note that terms and conditions apply. 


\title{
Probing background ionization: positive streamers with varying pulse repetition rate and with a radioactive admixture
}

\author{
S Nijdam ${ }^{1}$, G Wormeester ${ }^{2}, \mathbf{E}$ M van Veldhuizen ${ }^{1}$ and $\mathbf{U}_{\text {Ebert }^{1,2}}$ \\ ${ }^{1}$ Dept. Applied Physics, Eindhoven University of Technology, PO Box 513, 5600 MB Eindhoven, \\ The Netherlands \\ ${ }^{2}$ Centrum Wiskunde \& Informatica (CWI), PO Box 94079, 1090 GB Amsterdam, The Netherlands \\ E-mail: s.nijdam@tue.nl
}

Received 4 August 2011, in final form 26 September 2011

Published 28 October 2011

Online at stacks.iop.org/JPhysD/44/455201

\begin{abstract}
Positive streamers need a source of free electrons ahead of them to propagate. A streamer can supply these electrons by itself through photo-ionization, or the electrons can be present due to external background ionization. Here we investigate the effects of background ionization on streamer propagation and morphology by changing the gas composition and the repetition rate of the voltage pulses, and by adding a small amount of radioactive ${ }^{85} \mathrm{Kr}$.

We find that the general morphology of a positive streamer discharge in high-purity nitrogen depends on background ionization: at lower background ionization levels the streamers branch more and have a more feather-like appearance. This is observed both when varying the repetition rate and when adding ${ }^{85} \mathrm{Kr}$, though side branches are longer with the radioactive admixture. But velocities and minimal diameters of streamers are virtually independent of the background ionization level. In air, the inception cloud breaks up into streamers at a smaller radius when the repetition rate and therefore the background ionization level is higher. When measuring the effects of the pulse repetition rate and of the radioactive admixture on the discharge morphology, we found that our estimates of background ionization levels are consistent with these observations; this gives confidence in the estimates.

Streamer channels generally do not follow the paths of previous discharge channels for repetition rates of up to $10 \mathrm{~Hz}$. We estimate the effect of recombination and diffusion of ions and free electrons from the previous discharge and conclude that the old trail has largely disappeared at the moment of the next voltage pulse; therefore the next streamers indeed cannot follow the old trail.
\end{abstract}

(Some figures may appear in colour only in the online journal)

\section{Introduction}

Positive streamers require a source of electrons in front of the streamer head in order to propagate. These electrons can either be created by non-local photo-ionization from the streamer head itself, or they can already be present in the form of background ionization, either free or attached to an electronegative molecule like oxygen. Photo-ionization is a strong non-local mechanism in air that depends on the presence of both nitrogen and oxygen: fast electrons in the discharge excite nitrogen levels that subsequently emit ultraviolet (UV) photons that can then ionize oxygen. However, in past work [1], we have shown that even in nitrogen with impurity levels well below $1 \mathrm{ppm}$, positive streamers propagate at similar velocities as in air, but they are thinner, less straight and they branch more; the streamers can even exhibit a featherlike structure. The similarity of streamer velocities in air or high-purity nitrogen indicates that either low levels of oxygen still enable sufficient photo-ionization for a streamer to propagate or that the streamers can propagate by background ionization. Streamer simulations [2] actually have shown that even an oxygen level of $1 \mathrm{ppm}$ in nitrogen can support streamer 
propagation by photo-ionization at velocities similar to those in air, but that background ionization can support streamer propagation as well, and that this mechanism becomes more important at lower oxygen densities.

Here we focus on the effects of background ionization by varying its level. Under normal circumstances, there are two probable sources for background ionization: natural background ionization from cosmic radiation or radioactivity, and remaining ionization from previous discharges in a repetitive discharge mode. In the present experiments, we again largely vary the nitrogen : oxygen ratio to modify photoionization, and we now vary the repetition rate of the voltage pulse and we use a radioactive admixture to change the background ionization.

In air, electrons produced by any mechanism quickly attach to oxygen molecules. Therefore the ionization mostly consists of positive and negative ions. At the moderate electric fields outside the streamer head, these positive and negative ions are unable to create secondary ionization (and therefore an avalanche). Only when the local electric field is sufficiently high, e.g. close to a streamer head, can an electron detach from a negative oxygen atom, and it can then create an avalanche. In nitrogen of sufficiently high purity the recombination rate of electrons and ions can be higher than the attachment rate of electrons to oxygen molecules and therefore most electrons will remain free until recombination.

Natural background ionization levels by radioactivity and cosmic rays are normally around $10^{3}-10^{4} \mathrm{~cm}^{-3}$ in natural air at standard temperature and pressure ([3] and references therein). The actual level depends on many parameters, including building material type, ventilation, room or vessel dimensions and pressure. In our stainless steel vacuum vessel, the ionization levels will be lower than in ambient air at the same pressure-except, of course, with the radioactive admixture. This is because we use artificial air and highpurity nitrogen rather than ambient air (although the supplier did not specify the radon levels), the steel emits less radon than building materials and the vessel may shield part of the cosmic rays. In practice, it is very difficult to estimate the background ionization level inside the vessel, but it can be safely assumed to be lower than $10^{3} \mathrm{~cm}^{-3}$.

The level of background ionization caused by previous discharges in a mode of repetitive voltage pulses depends on the pulse repetition rate and on the streamer strength and density. At high streamer densities (when the streamer channels fill most of the discharge volume) a $1 \mathrm{~Hz}$ repetition rate can create a background ionization level of about $10^{7} \mathrm{~cm}^{-3}$ according to simple estimates by Pancheshnyi [3] as will be discussed in more detail in section 2 .

\subsection{Experimental results}

Hartmann and Gallimberti [4] have experimented with positive streamer discharges in a jet so that the remaining products of previous discharges are removed before the next discharge. They see a clear effect of the jet on streamer properties, but attribute this to the removal of nitrogen metastables and not of residual ionization.
Takahashi et al [5] have used a $\mathrm{KrF}$ laser to produce UV radiation of $248 \mathrm{~nm}$ wavelength which produced enhanced levels of background ionization in an atmospheric pressure argon discharge. They observe that in a $30 \mathrm{~mm}$ pointplane streamer discharge with a $20 \mathrm{kV}$ pulse, branching is suppressed significantly when the background ionization is above $5 \times 10^{5} \mathrm{~cm}^{-3}$. A similar result was found by Mathew et al [6] who have studied the effects of preionization (by $\mathrm{x}$-ray radiation) on the uniformity of discharges in $\mathrm{F}_{2}$ excimer laser gas mixtures $\left(\mathrm{He} / \mathrm{F}_{2}\right.$ mixtures with various ratios). They found that at preionization levels of $10^{7} \mathrm{~cm}^{-3} \mathrm{bar}^{-1}$ and higher the discharge was diffuse (uniform) and at lower preionization levels, the discharge became filamentary or streamer-like.

\subsection{Theoretical results}

The older literature $[7,8]$ offers a simple argument for why the exact source of electrons ahead of the streamer should not influence its properties too much, if electrons are available at all: as the electrons are exponentially multiplied in the active ionization region ahead of the streamer, where the electric field is above the breakdown value, the precise electron density at the front edge of the active zone should influence streamer properties only logarithmically.

Probably inspired by this argument, in early streamer simulations $[9,10]$ the photo-ionization in air was replaced by background ionization to ease the computational demands. Dhali and Williams [9] used a high uniform background ionization level of the order of $10^{5}-10^{8} \mathrm{~cm}^{-3}$, while Vitello et al [10] used a more realistic value of $10^{3} \mathrm{~cm}^{-3}$. Later, appropriate numerical means were made available to simulate photo-ionization, as reviewed, e.g. in [11].

The effects of different background ionization levels on streamers have been simulated recently by Bourdon et al [12] and by Wormeester et al [2]. Bourdon et al simulated both positive and negative streamers in preheated air where pulse repetition rates of $10-30 \mathrm{kHz}$ were approximated by homogeneous background ionization densities of up to $10^{9} \mathrm{~cm}^{-3}$ (see section 2 for such estimates). They found that the background ionization density has a small influence on negative streamers, but a significant influence on positive streamers; this agrees with earlier results by Luque et al [13] where the same asymmetry was found for the effect of photoionization on positive or negative streamers, and an explanation was given.

Wormeester et al found similar results and also studied different nitrogen:oxygen ratios. In that work, we show that photo-ionization and background ionization have roughly the same effect on streamer velocities, and that only a low contribution from either of these processes is required to ensure streamer propagation (e.g. background ionization larger than $10^{3} \mathrm{~cm}^{-3}$ ). For example, a concentration of $1 \mathrm{ppm}$ (parts per million) oxygen in nitrogen is enough to make a streamer propagate through photo-ionization, without the need for background ionization. In this case, the propagation velocity is about $20 \%$ lower than under similar conditions in air. A similar propagation velocity is found when photo-ionization is turned off and a background ionization level of $10^{7} \mathrm{~cm}^{-3}$ 
for positive and negative ions is assumed. However, streamers with strong photo-ionization as in air are thicker than with the weak photo-ionization of nitrogen with $1 \mathrm{ppm}$ oxygen.

In [2] we find that in air, at streamer repetition rates up to $1 \mathrm{kHz}$, the streamer propagation is dominated by photo-ionization, while in high-purity nitrogen, background ionization can already dominate at a pulse repetition rate of $1 \mathrm{~Hz}$. Finally, we see that our short streamers already branch in 'pure' nitrogen, but not yet in air under otherwise identical conditions.

We finally remark that all above simulations are performed by approximating the charged particles by their densities. But in [14], we have argued that the feathery structures in highpurity nitrogen are probably due to single electrons creating ionization avalanches. Resolving the avalanche density therefore requires an approach resolving single electrons. Such methods have recently been developed in [15-19].

\subsection{Discussion and approach of the paper}

We investigate here the importance of background ionization for streamer inception, propagation and structure by artificially increasing this background ionization. There are a few methods to increase ionization levels in gases. The simplest method is to use (corona) discharges. The effects of leftover ionization from these prior discharges on a new discharge can be investigated by changing the repetition rate of the voltage pulses. We have measured the effect of repetition rates between 0.01 and $10 \mathrm{~Hz}$. The results of these measurements and related topics are discussed in section 3 . If the repetition rate has a significant influence on streamer propagation, one could expect that streamers in subsequent discharges follow the pre-ionized paths of previous discharges. In section 4 we investigate this question both experimentally and theoretically.

Another means to artificially enhance background ionization in a gas is to add radioactive compounds. The alpha and/or beta particles created in the radioactive decay can ionize many more gas molecules, thereby increasing the background ionization. The advantage of this method is that the resulting ionization level on average is homogeneous in space and time. Disadvantages are that the gas mixtures with radioactive admixtures are expensive and require special handling. We have performed measurements on streamer discharges in nitrogen with a small addition of radioactive krypton-85. The results of these measurements are discussed in section 5. By comparing them with the effects of repetition rate, a consistent picture of the effects of background ionization levels on positive streamers is formed.

There are more methods to increase background ionization. For example irradiation with UV or x-rays. However, radiation that is still transmitted by the windows of our vessel (wavelengths above $\sim 170 \mathrm{~nm}$ ) is not able to directly ionize nitrogen or oxygen molecules. It can be used to liberate electrons from electrodes and walls or from impurities, but this is of limited use in our large vessel as it would lead to a very inhomogeneous distribution of ionization density. At higher intensities it is possible to ionize molecules or atoms with two or more photons but these high intensities make it impossible to produce homogeneous ionization levels throughout the vessel.
With special windows, it would be possible to use UV or x-ray radiation of lower wavelengths. However, both the installation of such windows as well as the generation of enough radiation of the right wavelengths would take a lot of effort. Furthermore, this would still result in a quite inhomogeneous distribution of background ionization with an unknown density. Takahashi et al [5] used a KrF laser to increase the background ionization in pure argon as was discussed above. They use a relatively small beam $(1 \mathrm{~cm}$ diameter), and therefore they have enough intensity to achieve two-photon ionization in part of their gas volume, while onephoton ionization is impossible at the wavelength they use.

More exotic methods like electron or ion beams to directly inject charges into the volume have to be discarded for similar reasons.

In addition to the nitrogen-krypton mixture discussed above, we use pure nitrogen (in two purity levels) and artificial air in our experiments. (Artificial) air is the gas mixture that is mostly used for streamer research and therefore makes a good benchmark, while the low oxygen levels in pure nitrogen suppress photo-ionization and therefore give more insight into the effects of background ionization. To better understand our experimental results, we have calculated the expected values of background ionization levels under various relevant conditions. The methods and results of these calculations will be given in section 2.1 .

\section{Ionization estimates and experimental conditions}

\subsection{Calculating background ionization levels}

Here we show the mechanisms that determine background ionization and give some rough estimates of the expected recombination times and ionization levels under different conditions. In particular, the recombination times are important as they determine the ionization levels from previous discharges, as well as the equilibrium level when a constant electron source is present (as in the case of a radioactive admixture).

2.1.1. Electron attachment. As streamer discharges produce high (local) ionization levels, they can increase the background ionization for subsequent discharges. In gas mixtures with high oxygen concentrations, electrons quickly attach to oxygen molecules $[3,20]$ :

$$
\begin{aligned}
& \mathrm{e}+\mathrm{O}_{2}+\mathrm{O}_{2} \rightarrow \mathrm{O}_{2}^{-}+\mathrm{O}_{2} \\
& k_{\text {att1 }}=2 \times 10^{-30} \mathrm{~cm}^{6} \mathrm{~s}^{-1}
\end{aligned}
$$

or, in gases with low oxygen concentrations:

$$
\begin{gathered}
\mathrm{e}+\mathrm{O}_{2}+\mathrm{N}_{2} \rightarrow \mathrm{O}_{2}^{-}+\mathrm{N}_{2} \\
k_{\mathrm{att} 2}=8 \times 10^{-32} \mathrm{~cm}^{6} \mathrm{~s}^{-1} .
\end{gathered}
$$

This is based on three-body attachment, which is only valid for low fields and high gas densities $\left(E / n_{0}<5 \mathrm{Td}[21,22]\right)$. Inside a streamer head fields will be significantly above this 
level, but after the streamer phase has passed, fields will be below it. The free electron density $n_{\mathrm{e}}$ will decrease as a function of time as

$$
\begin{aligned}
\partial_{t} n_{\mathrm{e}}= & -k_{\mathrm{att} 1} \cdot n_{\mathrm{e}} \cdot\left[\mathrm{O}_{2}\right]^{2} \\
& -k_{\mathrm{att} 2} \cdot n_{\mathrm{e}} \cdot\left[\mathrm{O}_{2}\right] \cdot\left[\mathrm{N}_{2}\right]
\end{aligned}
$$

if diffusion and drift are neglected. The attachment time $t_{\text {att }}$ (the e-folding time of the exponential decay) is determined by (3) as

$$
t_{\mathrm{att} 1}=\left(k_{\mathrm{att} 1} \cdot\left[\mathrm{O}_{2}\right]^{2}\right)^{-1}
$$

for air, or for oxygen concentrations below a few per cent:

$$
t_{\mathrm{att} 2}=\left(k_{\mathrm{att} 2} \cdot\left[\mathrm{O}_{2}\right] \cdot\left[\mathrm{N}_{2}\right]\right)^{-1} .
$$

The attachment time can vary significantly depending on oxygen content and pressure: in air at standard temperature and pressure, $t_{\text {att } 1}$ is about $20 \mathrm{~ns}$ while in pure nitrogen with about $0.1 \mathrm{ppm}$ of oxygen contamination at $200 \mathrm{mbar}$, $t_{\mathrm{att} 2}$ is about $5 \mathrm{~s}$. The rates used here are based on cold gas with no electric field present. Therefore, the ratio between free electrons and negative ions will be many orders higher in air than in this 'pure' nitrogen.

2.1.2. Recombination reactions. In air, after electron attachment, two- and three-body ion-ion dissociative recombination determines the rate of plasma decay [3]:

$$
\begin{gathered}
\mathrm{O}_{2}^{-}+\mathrm{O}_{4}^{+} \rightarrow \mathrm{O}_{2}+\mathrm{O}_{2}+\mathrm{O}_{2} \\
k_{\mathrm{rec} 1}=10^{-7} \mathrm{~cm}^{3} \mathrm{~s}^{-1}, \\
\mathrm{O}_{2}^{-}+\mathrm{O}_{4}^{+}+\mathrm{M} \rightarrow \mathrm{O}_{2}+\mathrm{O}_{2}+\mathrm{O}_{2}+\mathrm{M} \\
k_{\mathrm{rec} 2}=2 \times 10^{-25} \mathrm{~cm}^{6} \mathrm{~s}^{-1},
\end{gathered}
$$

where $\mathrm{M}$ denotes either a neutral oxygen or a neutral nitrogen molecule. The $\mathrm{O}_{4}^{+}$in these reactions is created quickly after the discharge from reactions of $\mathrm{N}_{2}^{+}$to $\mathrm{N}_{4}^{+}, \mathrm{O}_{2}^{+}$and finally $\mathrm{O}_{4}^{+}$, see e.g. [23]. The effective recombination rate for oxygen : nitrogen mixtures is therefore

$$
k_{\text {rec-air }} \approx k_{\text {rec } 1}+k_{\text {rec } 2} \cdot[\mathrm{M}] .
$$

For pure nitrogen (i.e. without electron attachment), we use $k_{\text {rec- } \mathrm{N}_{2}}=5 \times 10^{-7} \mathrm{~cm}^{3} \mathrm{~s}^{-1}$, which, at room temperature, is equal to $k_{\text {rec-air }}$ at a pressure of about $80 \mathrm{mbar}$ ([M] $=$ $\left.2 \times 10^{18} \mathrm{~cm}^{-3}\right)$. This effective recombination rate includes many different reactions.

2.1.3. Homogeneous recombination. If spatial inhomogeneity, diffusion and the drift of charged particles are neglected, the ionization density $n$ in a neutral plasma with $n=n_{+}=n_{-}$ decreases as a function of time $t$ as

$$
\partial_{t} n=-k_{\mathrm{rec}} \cdot n^{2}
$$

here $t$ is time, and $k_{\text {rec }}$ is the effective recombination rate for given gas composition and density, as described above. Equation (9) is solved by

$$
n(t)=\frac{n(0)}{1+n(0) k_{\mathrm{rec}} t},
$$

where $n(0)$ is the ionization density at time 0 ; for a streamer under standard conditions it is about $10^{13}-10^{14} \mathrm{~cm}^{-3}$. For all practical values of $k_{\text {rec }}$, for typical values of $n(0)$ in a fresh streamer and for timescales above milliseconds this equation is well approximated by

$$
n(t)=\frac{1}{k_{\mathrm{rec}} t},
$$

i.e. it is independent of the initial ionization density. This means that $1 \mathrm{~s}$ after a discharge, the ionization density in air at atmospheric pressure will be roughly $2 \times 10^{5} \mathrm{~cm}^{-3}$, when homogeneity is assumed. At 0.1 and $10 \mathrm{~s}$ after a discharge, the ionization density will be ten times as high or low, respectively. At other pressures, this number will be different, according to equation (8). Therefore, in air, at 200 and 25 mbar, the ionization density $1 \mathrm{~s}$ after a discharge will be $9 \times 10^{5}$ and $4 \times 10^{6} \mathrm{~cm}^{-3}$ respectively.

In pure nitrogen (with less than $1 \mathrm{ppm}$ oxygen concentration), the attachment time will always be much longer than the recombination time. Therefore, electrons will mostly recombine before they attach to oxygen. This means that the negative charges in pure nitrogen will mostly consist of free electrons, while in air they are mostly negative ions.

Note that the reactions above are relevant for nitrogenoxygen mixtures at pressures close to atmospheric. At much lower and higher pressures and in other gases, other mechanisms start to dominate the recombination reactions. For example, at pressures relevant for sprite discharges (below 1 mbar), dissociative attachment and recombination become very important loss mechanisms [24]. Furthermore, we assume that the discharge occurs in an environment where bulk gas recombination is the major ionization loss process. This means that the vessel or room dimensions have to be sufficiently large to neglect recombination at the wall, and that there should be no (strong) gas flow which can refresh the gas between repetitive pulses.

2.1.4. Recombination and diffusion. The calculation above assumes that the ionization is distributed homogeneously, and that it only changes by recombination. However, as the ionization by streamers is produced in narrow channels, diffusion will have an influence on ionization levels as well. When we include diffusion, equation (9) changes into

$$
\partial_{t} n=D_{\text {ion }} \cdot \nabla^{2} n-k_{\text {rec }} \cdot n^{2}
$$

with $D_{\text {ion }} \approx 5 \times 10^{-2} \mathrm{~cm}^{2} \cdot \mathrm{s}^{-1}$ the diffusion coefficient under standard conditions in air [3, 25, 26]. The diffusion coefficient scales inversely with pressure. In section 4 , we will evaluate equation (12) and show that the more localized ionization distribution in the trails of the streamers will create lower final ionization densities than obtained from the homogeneous estimate (11).

2.1.5. Constant ionization sources. There can be a constant source of ionization $S$ in a gas; this source can be natural radioactivity, cosmic rays or a radioactive admixture. 
Table 1. Gas impurity levels of relevant gases as provided by the gas supplier. Impurity levels are given as upper limits, except for ${ }^{85} \mathrm{Kr}$, which is dosed on purpose in the nitrogen/krypton mixture. Levels are in parts per million (ppm), n.s. indicates that no number is specified by the supplier.

\begin{tabular}{lllllllll}
\hline Gas & $\mathrm{N}_{2}$ & $\mathrm{O}_{2}$ & ${ }^{85} \mathrm{Kr}$ & $\mathrm{Ar}$ & $\mathrm{H}_{2} \mathrm{O}$ & $\mathrm{CO}_{2}$ & $\mathrm{CO}$ & $\mathrm{C}_{n} \mathrm{H}_{m}$ \\
\hline Nitrogen 6.0 & $\approx 100 \%$ & 0.2 & n.s. & n.s. & 0.5 & 0.1 & 0.1 & 0.1 \\
Nitrogen 7.0 & $\approx 100 \%$ & 0.03 & n.s. & n.s. & 0.05 & 0.03 & 0.03 & 0.03 \\
Artificial air & $\approx 80 \%$ & $\approx 20 \%$ & n.s. & 0.2 & 0.5 & 0.1 & 0.1 & 0.1 \\
Nitrogen/krypton & $\approx 100 \%$ & 0.5 & 0.0099 & 5 & 0.5 & 1 & 1 & 0.5 \\
\hline
\end{tabular}

Assuming again homogeneity, equation (12) has to be extended to

$$
\partial_{t} n=S-k_{\mathrm{rec}} \cdot n^{2} \text {. }
$$

In steady state $\left(\partial_{t} n=0\right)$, the production rate equals the recombination rate, and the equilibrium ionization density is

$$
n=\sqrt{\frac{S}{k_{\mathrm{rec}}}} .
$$

Again, the major negative charge carrier of the (quasi-neutral) ionization is very dependent on oxygen concentrations: at high concentrations (e.g. air) it is dominated by $\mathrm{O}_{2}^{-}$while in 'pure' nitrogen it is dominated by free electrons.

\subsection{Experimental conditions}

All experiments presented here were performed on positive streamers in a $50 \times 50 \mathrm{~cm}$ (diameter $\times$ height) vacuum vessel with a $160 \mathrm{~mm}$ separation point-plane geometry. This vessel is specifically designed for operation with high-purity gases at pressures between 25 and 1000 mbar. More details about this vessel can be found in [1].

For our experiments we have used four different gases: pure nitrogen in two qualities, artificial air and a mixture of (somewhat less) pure nitrogen with a small addition of radioactive krypton-85 (see section 5 for more details). Specifications of the gas mixtures according to the suppliers are given in table 1. During use, the gas inside the set-up is flushed with such a flow rate that all gas is replaced every $25 \mathrm{~min}$. All experiments are performed at room temperature. We have not found any significant differences in streamer properties (diameter, velocity and morphology) between the two different qualities of pure nitrogen.

To create high-voltage pulses we have used two power supplies, the so-called C-supply and the Blumlein pulser. The C-supply consists of a capacitor that is discharged by means of a sparkgap and thereby creates a voltage pulse with exponential rise and decay. Risetimes of the C-supply are typically of the order $100 \mathrm{~ns}$ and decay times are of order $5 \mu \mathrm{s}$ in the presented measurements. More details about the C-supply can be found in [27]. The Blumlein pulser consists of two $10 \mathrm{~m}$ long coaxial cables that are charged and then discharged by means of a multiple sparkgap. This creates a more or less rectangular voltage pulse with a length of about $130 \mathrm{~ns}$ and a risetime of about $10 \mathrm{~ns}$. This circuit is treated in more detail in [1]. We use two different circuits for two reasons: one is to compare with previous measurements performed with these two circuits. The second reason is that they both have their own advantages: the short pulse from the Blumlein pulser ensures that a rather constant background field is applied during a well-defined time interval in which the streamer propagates, and that it is turned off rapidly thereafter, while the long pulse of the C-supply can create very thin and long streamers.

The discharges are imaged by a Stanford Computer Optics 4QuikE ICCD camera with a Nikkor UV $105 \mathrm{~mm} f / 4.5$ lens. In the images presented here, the original brightness is indicated by the multiplication factor Mf, similar to what Ono and Oda introduced in [28] and as described by us in [1]. We have normalized the Mf value in such a way that the brightest image presented in [1] has an Mf value of 1. This has resulted in the following equation for the Mf value:

$$
\mathrm{Mf}=500 \cdot \mathrm{e}^{\frac{V_{\mathrm{g}}}{68.9 \mathrm{~V}}} \cdot \frac{1}{D^{2} \cdot\left(C_{\max }-C_{\min }\right)},
$$

with $V_{\mathrm{g}}$ the gain voltage of the camera, $D$ the inverse aperture (e.g. 4.5 for $f / 4.5$ ) and $C_{\max }$ and $C_{\min }$ the maximum and minimum count values of a pixel used in the image representation. The factor 500 comes from the normalization procedure and the camera gain factor of $68.9 \mathrm{~V}$ is a property of our camera. This calculation is only valid for images from this specific camera together with our set-up (lenses and windows) and a specific false-colour palette. Therefore our exact definition is different from the one by Ono and Oda [28] although the general concept is the same.

Note that we improved our fitting procedure of the camera calibration data since publication of $[1,29]$. Therefore the data presented there has a somewhat different definition of the Mf value. In the old definition, we used a camera gain factor of $58.4 \mathrm{~V}$ and a normalization value of 99 instead of 500. This means that images with Mf values around 1 will be virtually the same in [1] and in this work, but that high Mf values can be up to a factor of 2 lower in this work than in [1].

\section{Effects of repetition rate}

Both pulsed power supplies allow repetition rates of about $10 \mathrm{~Hz}$ and slower. Therefore we have measured the effect of the repetition rate on streamer morphology in high-purity nitrogen and in artificial air at 10, 1, 0.1 and $0.01 \mathrm{~Hz}$.

According to the estimates above, the repetition rate of $0.01 \mathrm{~Hz}$ will create a background ionization level of about $2 \times 10^{4} \mathrm{~cm}^{-3}$ at $200 \mathrm{mbar}$. This is close to natural background ionization levels. Therefore we do not expect to see many differences between a completely 'fresh' gas fill, and a gas fill $100 \mathrm{~s}$ after a streamer discharge. We will see that the higher repetition rates yield measurable effects. 


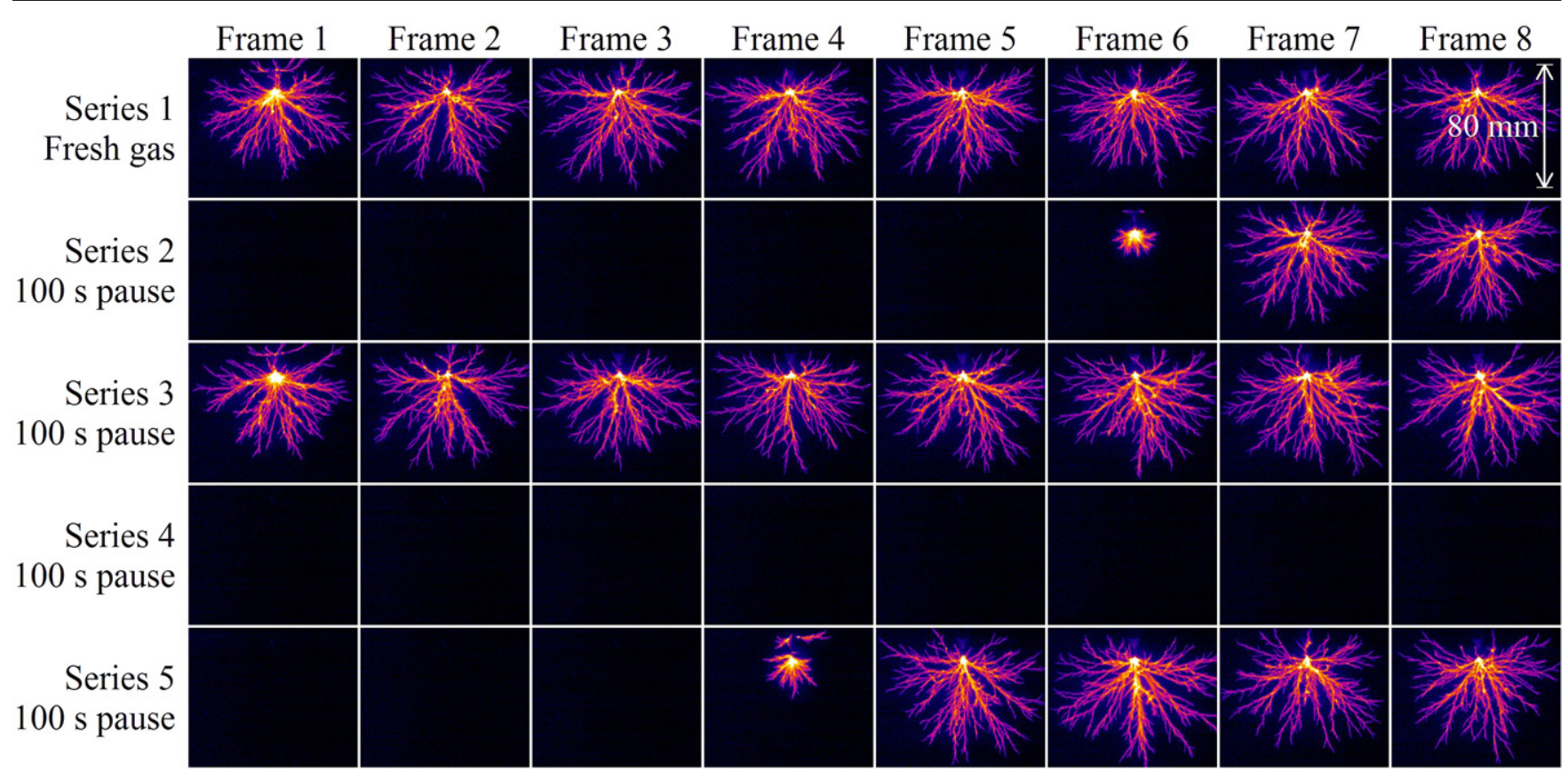

Figure 1. Five separate series of eight consecutive frames of streamer discharges (or attempts) in high-purity nitrogen. The series start at the first pulse of a 'fresh' gas filling. In series 1 this is a completely new fill of the vacuum vessel (it was pumped down to $2 \mathrm{mbar}$ before filling). In the other series no pulse was applied for at least $100 \mathrm{~s}$ prior to frame 1 . The frames are recorded in nitrogen 7.0 at $200 \mathrm{mbar}$ with $25 \mathrm{kV}$ positive pulses from the Blumlein pulser at a repetition rate of $1 \mathrm{~Hz}$. The Mf-value of the images is about 43 . The five series shown comprise the full measurement series.
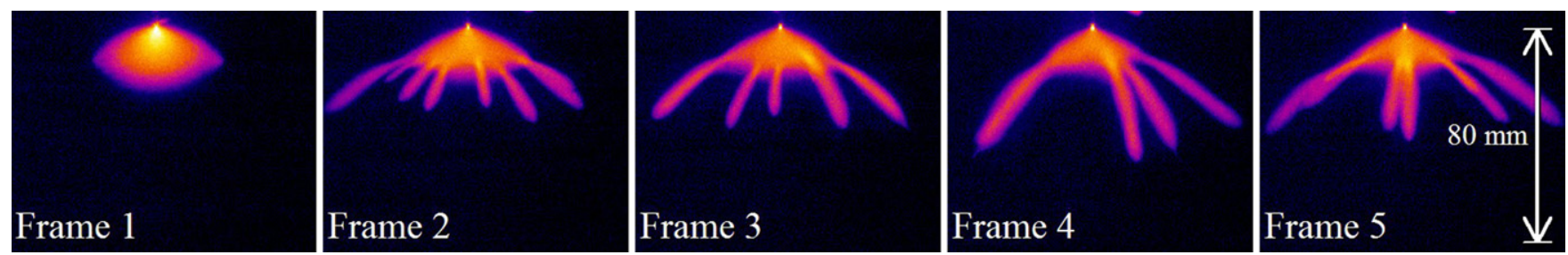

Figure 2. The first five frames of streamer discharges after a $100 \mathrm{~s}$ pause in artificial air. The frames are recorded at $200 \mathrm{mbar}$ with $25 \mathrm{kV}$ positive pulses from the Blumlein pulser at a repetition rate of $1 \mathrm{~Hz}$. The Mf-value of the images is about 190.

\subsection{General results}

\subsubsection{Inception, first discharge and subsequent discharges.} Streamer discharges in 200 mbar pure nitrogen with $25 \mathrm{kV}$, $130 \mathrm{~ns}$ pulses from the Blumlein pulser often do not initiate at a pulse repetition rate of $0.01 \mathrm{~Hz}$. Therefore we have used a different method to investigate streamer propagation at this repetition rate. After a $100 \mathrm{~s}$ pause (no pulses), we switch the pulse source on with a repetition rate of $1 \mathrm{~Hz}$. ICCD camera frames of the first 10-30 pulses are then captured and stored. In this way, the first pulse which initiates a streamer as well as some subsequent discharges are nearly always captured. In one case, we have taken this approach a bit further and have used a completely fresh gas fill to start with, instead of a 100 s pause. In this case, we first pumped the vacuum vessel down to $2 \mathrm{mbar}$, before refilling it to $200 \mathrm{mbar}$. The results of these measurements are shown in figure 1 for pure nitrogen and figure 2 for artificial air.

In pure nitrogen we can observe that indeed the streamers do not always initiate on the first pulse under the specified conditions. In only two of the five attempts did the streamers initiate on the first pulse. One of these two attempts was the case when nearly all of the gas in the vessel was replaced (a fresh gas fill). In another attempt (series 4), no inception at all was observed during the first ten pulses. In general we observe an inception probability of the order of $10 \%$ under these conditions. In all attempts that show inception, the morphology of the first discharge is different from subsequent discharges. The propagation length is usually shorter and there seems to be a denser core of the streamer tree near the tip (see also next section).

In air inception is easier and (for these conditions) usually occurs at the first pulse as is shown in figure 2. However, we again see that the first discharge after $100 \mathrm{~s}$ waiting is different from subsequent discharges. In the first discharge, only an inception cloud is visible, while in subsequent discharges, this inception cloud is smaller and streamers emerge from it. It appears that the inception cloud of the second frame is still larger than the inception cloud of subsequent frames. We have observed the same behaviour in all three measurement series we have recorded under these conditions.

3.1.2. Dependence on repetition rate. We have measured the effect of the pulse repetition rate with repetition rates from 

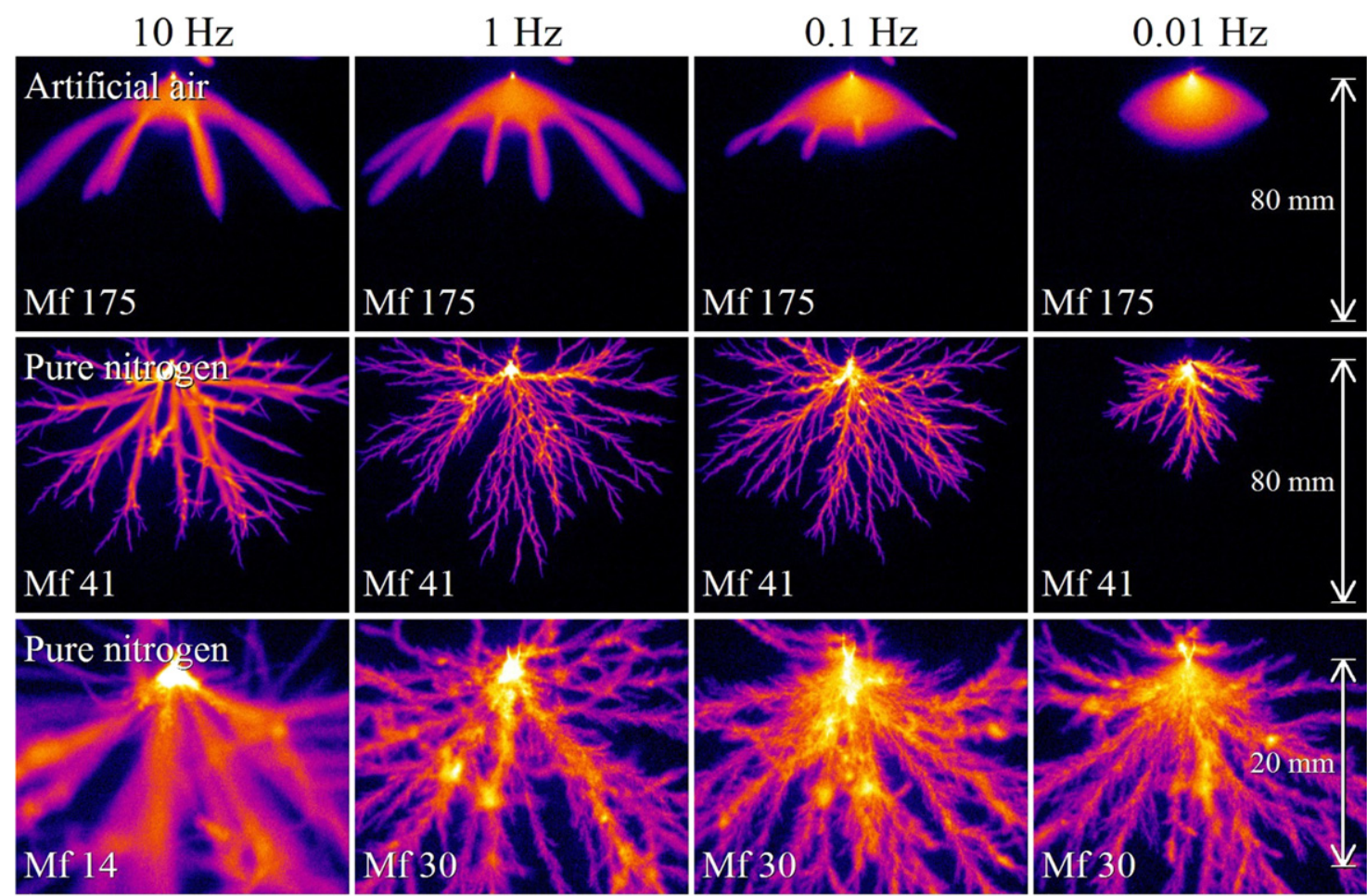

Figure 3. Overview (top and middle row) and zoomed (bottom row) images of the effects of pulse repetition rate on streamer morphology at 200 mbar. These images are acquired in artificial air and nitrogen 7.0 with the Blumlein pulser (pulse length $130 \mathrm{~ns}$ ) at $25 \mathrm{kV}$. The $0.01 \mathrm{~Hz}$ images are created similarly to the ones from figure 1 . Shown here is the first image with visible streamers.

$10 \mathrm{~Hz}$ down to $0.01 \mathrm{~Hz}$ in pure nitrogen and in artificial air. At least four pulses with the specified repetition rate have occurred before capturing the images that are shown in figure 3. Again, the measurements at $0.01 \mathrm{~Hz}$ have been performed differently as discussed above, and the first image which shows a streamer discharge is plotted. Except for pure nitrogen at $0.01 \mathrm{~Hz}$, all conditions had a near $100 \%$ inception probability.

Figure 3 shows that in both gases the streamer morphology depends on the repetition rate. In all cases, the length of the streamers is limited by the short pulse duration $(\approx 130 \mathrm{~ns})$ of the Blumlein pulser.

In pure nitrogen, the inception cloud is very small (as is always the case). Here the main visible effect is that for lower repetition rates the streamers become thinner, the number of streamers increases and they get an increasingly feather-like structure (see also [1]). The bright core that is visible in the first successful discharges in figure 1 and in the 0.1 and $0.01 \mathrm{~Hz}$ overview images in figure 3 consists of many overlapping feather-like streamers, as can be seen in the zoomed images in figure 3. A more quantitative description of the feathers is given in section 5.3 and figure 12. The total length (and therefore the average propagation velocity) does not vary much between 10 and $0.1 \mathrm{~Hz}$. At $0.01 \mathrm{~Hz}$, the streamers are shorter and show a large variation in size, but this can probably be attributed to late inception. As was shown in the previous section, streamers mostly do not initiate on the first pulse after a $100 \mathrm{~s}$ pause. Therefore we suggest that the first streamer discharge that does initiate, does so at a random moment during the pulse. This means that the length of the streamer discharge at $0.01 \mathrm{~Hz}$ is not a good indication for its propagation velocity, but rather for the actual inception time within the voltage pulse. Except for the difference in discharge length, the general morphology is the same for 0.1 and $0.01 \mathrm{~Hz}$.

In air, we see that the inception cloud becomes larger for lower repetition rates. Simultaneously, the streamers emitted from this cloud become shorter and thinner. At $0.01 \mathrm{~Hz}$, there is only a hint of a streamer coming from this cloud (on the right-hand side in this example).

\subsection{Discussion}

We have observed that at higher pulse repetition rates, there are fewer but thicker streamer channels, both in artificial air and in high-purity nitrogen; and they branch less. This effect is more prominent close to the electrode tip than at the outer edges of the streamer discharge. We have not observed any difference in streamer morphology between repetition rates of $0.1 \mathrm{~Hz}, 0.01 \mathrm{~Hz}$ and discharges in a fresh gas fill. However, in pure nitrogen, streamers do initiate more easily at $0.1 \mathrm{~Hz}$ than at $0.01 \mathrm{~Hz}$. In artificial air, the inception cloud is larger at lower repetition rates.

That streamers branch less at higher repetition rates and consecutively higher background ionization was observed already by Pancheshnyi [3] and is in accordance with the observations described above and with observations by others (e.g. $[5,30])$. Conceptually, this observation is consistent with the fact that streamers are wider and more stable in air than in pure nitrogen: in air, photo-ionization supplies a much higher level of ionization in the proximity of the streamer head. This is illustrated in figure 4 (taken from [2]) that shows the electron density on the axis of a simulated streamer in three different gases: in pure nitrogen without photo-ionization, but with a 


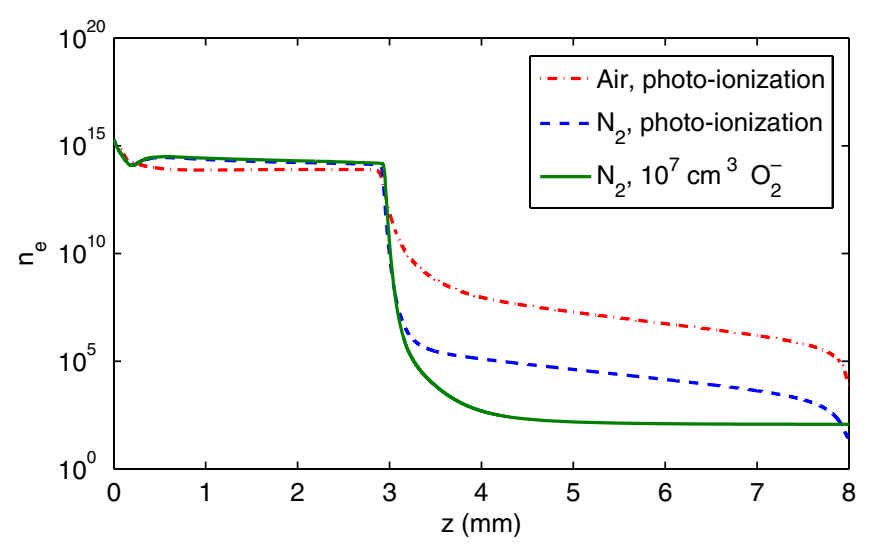

Figure 4. Simulated electron densities on the streamer axis. The streamers propagate from left to right with their heads at about $z=3 \mathrm{~mm}$. The solid curve shows nitrogen with a background ionization of $10^{7} \mathrm{~cm}^{-3}$, but without photo-ionization, the dashed curve shows nitrogen with the photo-ionization corresponding to an admixture of 1 ppm oxygen, and the dashed-dotted curve shows air with its corresponding photo-ionization. A similar figure was discussed and published by Wormeester et al [2].

background ionization of $10^{7} \mathrm{~cm}^{-3}$, in nitrogen with the photoionization corresponding to an admixture of $1 \mathrm{ppm}$ oxygen, and in air with its photo-ionization. Air has by far the highest ionization profile ahead of the streamer. The conclusion is that a high ionization density ahead of the streamer makes it propagate in a more stable manner, as different progression of parts of the streamer front is averaged out by lateral diffusion and photo-ionization if applicable.

In air, the many electrons ahead of the streamer will form so many ionization avalanches in the active streamer ionization zone that they cannot be distinguished visually, but appear as a thick smooth streamer body. In contrast, in very pure nitrogen at low pulse repetition rates, avalanches created by single electrons are so few in number, that they can be distinguished visually according to estimates presented in [14]. We have suggested that these avalanches can be identified with the hairs in the feathery streamer structures close to the needle electrode as described in [1,14]; these 'feathers' can also be seen in the zoomed images at 0.1 and $0.01 \mathrm{~Hz}$ in figure 3. At higher repetition rates, the background ionization level increases, and the streamer propagation becomes more similar to that in air. As the streamers are denser close to the needle electrode, in a repetitive mode the background ionization density is higher there, and the streamers are wider and more stable close to the needle than at the outer edges of the discharge. This can also be verified in the figures.

In very pure nitrogen in the steel vessel at repetition rates of $0.01 \mathrm{~Hz}$, the background ionization is so low that sometimes no inception occurs within a $25 \mathrm{kV}$ voltage pulse of $130 \mathrm{~ns}$ duration. This observation might allow conclusions on the background ionization which will be worked out in the future.

The fact that we observe much easier inception in artificial air than in pure nitrogen seems to contradict the results of van Veldhuizen and Rutgers [31]. They found that ambient air, dry air and pure nitrogen exhibit roughly similar inception probabilities as a function of voltage. However, this could be due to the purity of their nitrogen being three orders of magnitude less than ours, as it was specified as 4.0, while we use 7.0 nitrogen. Furthermore, they triggered the discharge in a somewhat unsystematic manner manually every 10-60 s [32].

We have argued that at low background ionization densities and low photo-ionization, avalanches created by individual electrons can be visible. We cannot decide at the moment whether they stay in the avalanche phase (in that case they only run backwards towards the parent streamer channel), or whether they eventually develop their own space charge and become streamer branches and run forward. Only in the second case one could speak of streamer branching. We stress that streamers require sufficient net charge to support their propagation; this could explain why the 'feathers' stay short.

In contrast, if streamers appear visually smooth in air or at high repetition frequencies, one cannot conclude that the fluctuations due to the discreteness of electrons do not play a role. Luque and Ebert [19] have shown recently that the branching of positive streamers in ambient air is accelerated when the discreteness of electrons is taken into account, and that the predicted branching rate agrees with experimental observations.

Finally, we stress an apparent paradox: if ionization density is smoother at higher repetition frequencies, particle fluctuations should be less and instabilities should set in later. Therefore, one would expect that with a higher repetition frequency, the inception cloud around the needle electrode in the top row of figure 3 would become larger before it destabilizes and breaks up into streamers, but the opposite is the case. We have no explanation for this observation yet, except for the hypothesis that the quite thick streamers leave some macroscopic perturbations of the medium behind even after $1 \mathrm{~s}$. These could be ionization trails or thermal expansion and convection $[33,34]$.

\section{Repetition of streamer paths}

\subsection{General results}

If positive streamers in pure nitrogen propagate due to the background ionization left by previous discharges, one could expect that streamers in consecutive discharges would follow the same paths.

To check this hypothesis, images of consecutive discharges at $10 \mathrm{~Hz}$ repetition rate are plotted in figure 5. The bottom row of this figure shows overlays of three subsequent discharges that are coloured in red, green and blue. The images show that, in general, subsequent streamers do not follow the same path. Only some channels (outside the crowded central area) are rendered cyan, yellow or white, while most channels are either red, green or blue. If we assume that the streamer paths in subsequent voltage pulses are independent of each other, and if we take into account that the 2D images are projections of a 3D streamer tree, then we expect similar pictures. Therefore we can conclude that under the conditions of figure 5, consecutive discharges do not follow the old streamer trail.

In the bottom half of the discharge, the average separation between the channels in one image is over $30 \mathrm{~mm}$ if we assume 

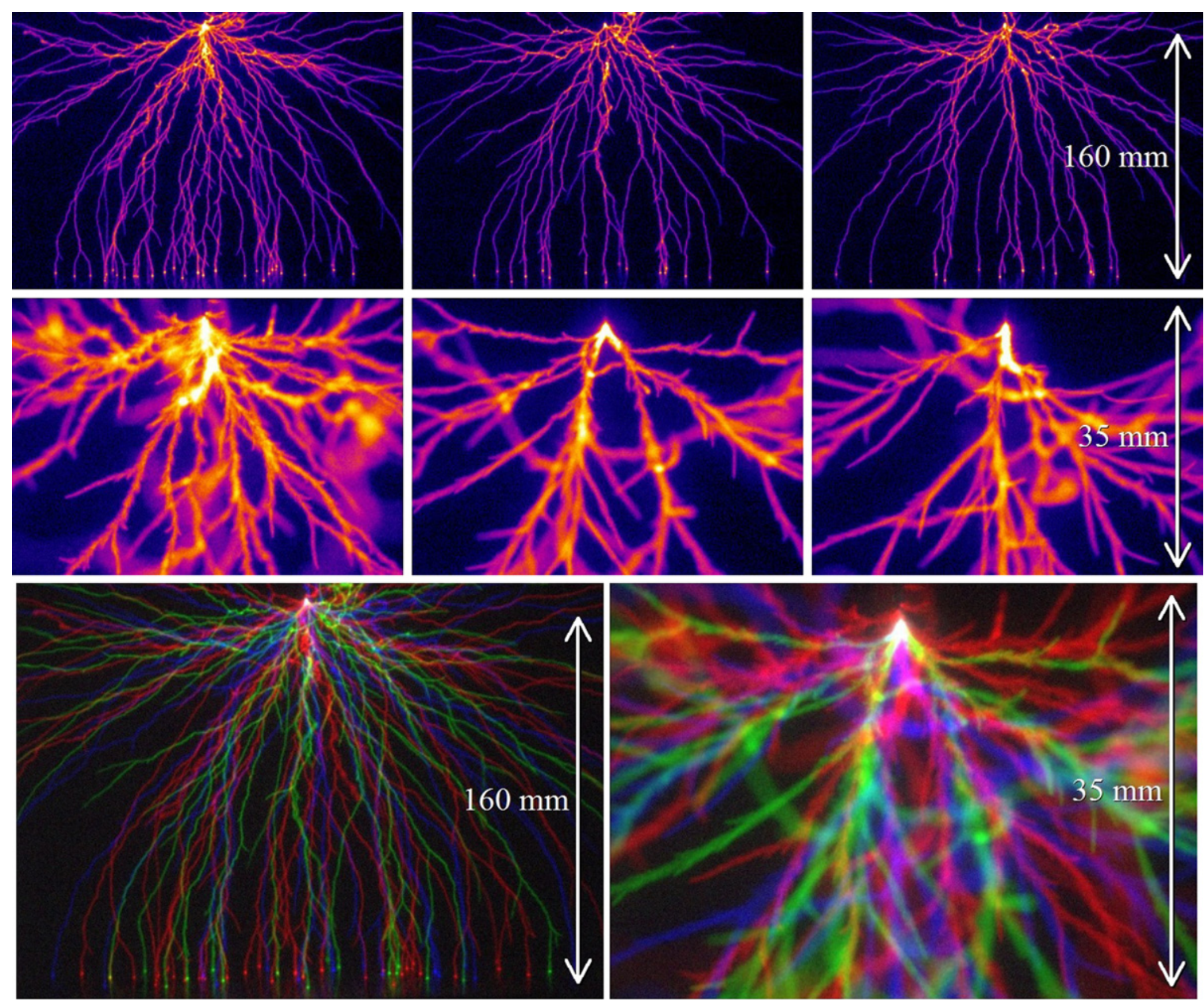

Figure 5. Two series of three consecutive images in nitrogen in overview (top row) and zoomed (middle row), both under similar conditions and with $10 \mathrm{~Hz}$ repetition rate. In the bottom row, the three consecutive images from the first or second row are coloured in red, green and blue, respectively, and overlaid. Therefore repetitive streamer paths are rendered yellow (red + green), cyan (green + blue) or white (red + green + blue), while single streamer paths are rendered red, green and blue. All images are acquired in 200 mbar nitrogen 6.0 with the C-supply at $15 \mathrm{kV}$. In both series, the first three discharge pulses after a break of about $10 \mathrm{~s}$ are shown.

cylindrical symmetry. This value is used as input for the calculations presented below.

As said in section 2.2, the linear dimensions of the vessel are $50 \mathrm{~cm}$ and the gas is renewed every $25 \mathrm{~min}$. The average gas flow velocity is therefore $300 \mu \mathrm{m} \mathrm{s}^{-1}$, i.e. the gas displaces on average by $30 \mu \mathrm{m}$ between voltage pulses at a repetition rate of $10 \mathrm{~Hz}$. This is $1 / 10$ of the minimal streamer diameter in pure nitrogen at 200 mbar according to [1], and therefore negligible.

\subsection{Channel repetition}

We now calculate the effects of recombination and diffusion on the ionization trail that is left by a previous streamer in pure nitrogen, correcting the estimates given earlier in section 2.1 . In this calculation we neglect space charge and fields and assume that the plasma will be electrically neutral soon after the external electric field has disappeared. We remark that most ionization anyhow resides in the electrically neutral interior of the streamer channel, and that only a small positive charge surplus is in the streamer skin [35]. We approximate the experimental conditions in nitrogen at 200 mbar by assuming that the streamer leaves an ionized trail behind that has a
Gaussian profile with a full-width at half-maximum (FWHM) of $300 \mu \mathrm{m}$ and a maximum ionization density of $10^{14} \mathrm{~cm}^{-3}$. In 200 mbar pure nitrogen, the ion diffusion coefficient is $D_{\text {ion }} \approx 0.25 \mathrm{~cm}^{2} \mathrm{~s}^{-1}$ and the recombination rate $k_{\text {rec-N }} \approx$ $5 \times 10^{-7} \mathrm{~cm}^{3} \mathrm{~s}^{-1}$. As already discussed in section 2.1 , most electrons stay free until they recombine with the ions. A brief estimate of charged particle densities and resulting electric fields shows that the electrons cannot diffuse independently of the ions. Rather, the joined diffusion of electrons and ions has to be modelled by ambipolar diffusion. The ambipolar diffusion coefficient is equal to

$$
D_{\mathrm{a}}=D_{\text {ion }}\left(1+\frac{T_{\mathrm{e}}}{T_{\mathrm{i}}}\right) \text {. }
$$

We assume that electron and ion temperatures are roughly equal, which means that $D_{\mathrm{a}} \approx 0.5 \mathrm{~cm}^{2} \mathrm{~s}^{-1}$ and use this number instead of $D_{\text {ion }}$ in our calculations.

We now solve $n(t, r)$ in equation (12) numerically. For simplicity, we assume that the streamers are distributed in a hexagonal grid with a distance of $30 \mathrm{~mm}$ to their neighbours, which can easily be approximated by a single streamer in 

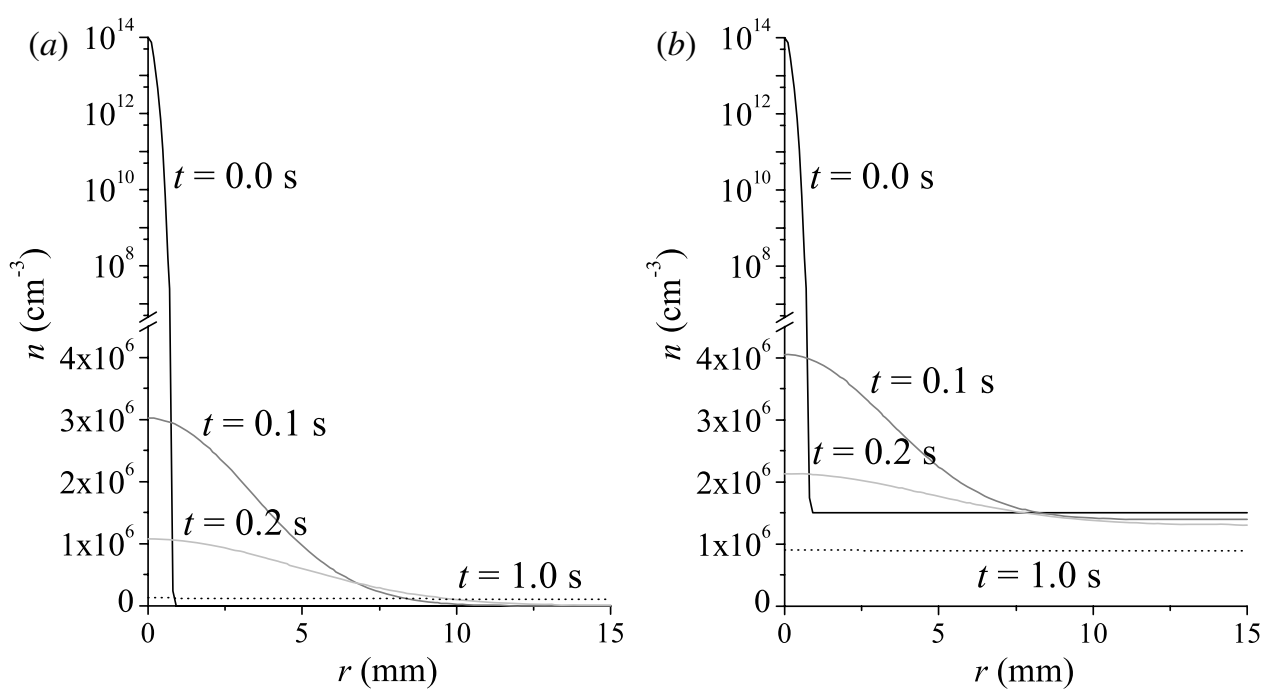

Figure 6. Ionization density as a function of distance $r$ to the centre of a streamer channel for different times $t$ after the discharge. Results are for pure nitrogen at $200 \mathrm{mbar}$ with $30 \mathrm{~mm}$ distance to the next streamer. Initial conditions include a background ionization level $n_{\text {ini }}=1 \times 10^{3} \mathrm{~cm}^{-3}$ for $(a)$ and $n_{\text {ini }}=1.5 \times 10^{6} \mathrm{~cm}^{-3}$ for $(b)$, both with the same central Gaussian streamer trail at $r=0$ $\left(\mathrm{FWHM}=0.3 \mathrm{~mm}\right.$ and $\left.n_{\text {channel }}=10^{14} \mathrm{~cm}^{-3}\right)$. Note that the vertical axis changes from a linear to a logarithmic scale at $n=5 \times 10^{6} \mathrm{~cm}^{-3}$.

cylindrical symmetry with $\partial n / \partial r=0$ as boundary condition (see the illustration in [35]). The initial conditions include the Gaussian streamer channel around $r=0$ together with a homogeneous background ionization level

$$
n(0, r)=n_{\text {ini }}+n_{\text {channel }} \cdot \mathrm{e}^{-\frac{r^{2}}{\sigma^{2}}}
$$

where $n_{\text {ini }}$ is the background ionization level, $n_{\text {channel }}$ is the maximal ionization density left at the centre of the channel and $\sigma$ is a measure for the width of the channel $(\sigma=$ FWHM $/ 2 \sqrt{\ln 2}$ ).

First we study the case where the initial background level is $n_{\text {ini }}=10^{3} \mathrm{~cm}^{-3}$, representative for the first discharge in virgin gas (figure $6(a)$ ). In this case, the ionization density at $t=0.1 \mathrm{~s}$ at the centre of the original channel $(r=0)$ is about 2700 times higher than on the edge of the domain $(r=15 \mathrm{~mm})$. This means that the channel is still clearly present. However, its width has become quite large (FWHM $\approx 8 \mathrm{~mm}$ ), as the figure shows. One second after the discharge the profile has become much wider (FWHM $\approx 15 \mathrm{~mm}$ ) and the ratio between the centre and the edge levels has decreased to about 1.2.

To determine the background ionization level after many shots, we have searched for a value of $n_{\text {ini }}$ where the spatially averaged calculated ionization density $n_{\text {ave }}$ at $t=0.1 \mathrm{~s}$ (averaged over cylindrical coordinates) is equal to $n_{\text {ini }}$. We call this the equilibrium background ionization density, $n_{\mathrm{eq}}=$ $n_{\mathrm{ave}}=n_{\text {ini }}$. Under the given conditions, $n_{\mathrm{eq}} \approx 1.5 \times 10^{6} \mathrm{~cm}^{-3}$. The results for the ionization density distribution with $n_{\text {eq }}$ as the initial background level are given in figure $6(b)$. Here, at $t=0.1 \mathrm{~s}$ the ratio between centre and edge ionization density is only 2.9 and the FWHM of the trail is again about $8 \mathrm{~mm}$.

From these calculations, we can conclude that in a $10 \mathrm{~Hz}$ repetitive discharge in 200 mbar nitrogen, the ionization trail left behind by the previous discharge is quite weak. At its centre its ionization density is less than 3 times higher than in the periphery. The ionization density decreases by more than 7 orders of magnitude within $0.1 \mathrm{~s}$.
At lower repetition rates, the trail is even less prominent. At $1 \mathrm{~Hz}$, the equilibrium background ionization level is about $n_{\mathrm{eq}} \approx 4.3 \times 10^{5} \mathrm{~cm}^{-3}$. When keeping all other parameters the same as in the previous calculations, the centre-to-edge density ratio is about 1.03. One pulse (or second) later, the trail has virtually disappeared.

Recent 2D drift-diffusion calculations by Nikipelov et al [36] show that at the timescales of interest $(0.1-1 \mathrm{~s})$, the streamer channel in air at standard temperature and pressure can even have lower ionization densities than the periphery. They show that the abundance of reactive (excited) species in the plasma channel leads to higher effective recombination rates in the channel and therefore the density (at long timescales) can be lower than outside the channel.

Note that the equilibrium ionization densities $n_{\mathrm{eq}}$ as defined above are close to an order of magnitude lower than the ionization levels calculated with equation (11) for a homogeneous initial distribution. This is because at the streamer core the ionization density and therefore the recombination rate are higher than in a homogeneous distribution of the same total number of charged species. At 200 mbar nitrogen and $1 \mathrm{~Hz}$, the result from equation (11) is $n \approx 2 \times 10^{6} \mathrm{~cm}^{-3}$ while for the same conditions $n_{\mathrm{eq}} \approx$ $4 \times 10^{5} \mathrm{~cm}^{-3}$. At $10 \mathrm{~Hz}$ repetition rate, these values are $2 \times 10^{7} \mathrm{~cm}^{-3}$ and $n_{\mathrm{eq}} \approx 1.5 \times 10^{6} \mathrm{~cm}^{-3}$, respectively. However, the value of $n_{\text {eq }}$ depends on the assumptions for streamer channel width, channel separation and maximal streamer ionization density. For high streamer densities (due to wide channels or small channel separation), $n_{\mathrm{eq}}$ becomes equal to $1 /\left(k_{\mathrm{rec}} t\right)$.

For streamers in air, photo-ionization can increase ionization levels outside of the streamer channels during streamer propagation.

In [3], Pancheshnyi also solves equation (12) numerically, but not in the radial direction of the streamer as we do, but between planar electrodes (though he speaks about a needleto-plane geometry). In his approach, the ionization density 
inside the streamer channel immediately after the discharge is assumed to be present homogeneously in the whole volume. Furthermore, he uses a fixed source of ionization to account for ionization by cosmic rays and natural background ionization.

Based on our calculations, it seems doubtful whether such a homogeneous background ionization is a reasonable approximation for the remainders of previous discharges in air at repetition rates of $10-30 \mathrm{kHz}[37,12]$, since diffusion coefficients and recombination rates are similar to those in nitrogen. However, Celestin, Bourdon et al [37,12] use in their models a homogeneous background ionization based on the results of the calculations by Pancheshnyi.

\subsection{Discussion}

The experimental results presented in figure 5 illustrate our general experience that within a single series of subsequent voltage pulses with repetition rates of $10 \mathrm{~Hz}$ or less, streamers follow a new path on every new voltage pulse for air, pure nitrogen and many other gases and gas mixtures. There is no indication that they are in any way influenced by the shape of the previous discharge. Only if the gap is so short that only one streamer propagates on the symmetry axis of the experiment, will subsequent streamers follow the same path; examples of such behaviour are shown in $[38,1]$ and many other publications. But whenever multiple channels are observed, the streamers take a new path on each instance.

If streamers do not follow (nearly) the same path as in the previous discharge, this can be either because there is no preference for the previous path or because the streamers are actually repelled by the old discharge trail. The theoretical estimates developed for our experiment in the section above suggest that the trail of a previous discharge is only five times more ionized than the periphery, and furthermore smeared out over a diameter of $5 \mathrm{~mm}$ due to diffusion and recombination in 200 mbar nitrogen at a repetition rate of $10 \mathrm{~Hz}$. At lower repetition rates, the trail will be even less visible. Apparently, the small differences in ionization density remaining from the previous discharge have no influence on the path of the next discharge. When an increased recombination rate in the streamer interior is taken into account as suggested by Nikipelov et al [36], the old streamer path could even approach a lower ionization level than the surrounding and therefore become slightly repulsive.

\section{Addition of radioactive ${ }^{85} \mathrm{Kr}$}

In order to increase the background ionization density in pure nitrogen without changing the repetition rate, we have performed experiments with a radioactive admixture of krypton-85.

\subsection{The admixture and its ionization rates}

Krypton-85 $\left({ }^{85} \mathrm{Kr}\right)$ is a radioisotope of krypton with a halflife of 10.756 years. The most common decay (99.57\%) is by emission of a beta particle and formation of the stable isotope ${ }^{85} \mathrm{Rb}$. The emitted beta particles have a maximum energy of $687 \mathrm{keV}$ and an average energy of $251 \mathrm{keV}$. We will use the average energy for our calculations. Krypton- 85 has the advantage that it is an inert gas. Therefore it is used in many applications, e.g. in leak detection [39] or as a small admixture to argon as an ignition aid for high-intensity discharge (HID) lamps [40].

We use a mixture of $9.9 \mathrm{ppb}$ (parts per billion) of ${ }^{85} \mathrm{Kr}$ in pure nitrogen. Details of the impurities in this mixture as specified by the gas supplier are given in table 1 . This mixture has a specified decay rate of $500 \mathrm{~Bq} \mathrm{~cm}^{-3}\left(=500 \mathrm{kBql}^{-1}\right)$ at standard temperature and pressure, or about $13.5 \mathrm{nCi} \mathrm{cm}^{3}$ ( $\mathrm{nCi}=$ nanocurie $=37$ decays per second). In our vessel with a volume of about $100 \mathrm{~L}$, this leads to about $5 \times 10^{7}$ decays per second at atmospheric pressure. The average time between decays is $20 \mathrm{~ns}$ which means that during a streamer discharge of $100 \mathrm{~ns}-1 \mu$ s duration 5-50 decays will take place in our gas volume.

According to an empirical formula by Glendenin [41, 42], the penetration length of a $251 \mathrm{keV}$ beta particle in air at atmospheric pressure is about $50 \mathrm{~cm}$ which is nearly equal to the dimensions of our vacuum vessel. The average path length before an emitted particle hits the wall is about $25 \mathrm{~cm}$. Therefore we assume that only half of the energy of the particle is absorbed inside the gas.

When we assume that half of the energy loss of the particle inside the gas is used to ionize molecular nitrogen (ionization energy $15.6 \mathrm{eV}$ ), we can estimate the electronion pair production at about 4000 pairs per emitted beta particle. Combined with the decay rate given above, this gives an ion-electron pair production rate $S$ of about $2 \times 10^{6} \mathrm{~s}^{-1} \mathrm{~cm}^{-3}$.

We can use this production rate together with the recombination rate for pure nitrogen $k_{\text {rec- } \mathrm{N}_{2}}=5 \times$ $10^{-7} \mathrm{~cm}^{3} \mathrm{~s}^{-1}$ and equation (14) to calculate the equilibrium ionization density. At $1000 \mathrm{mbar}$ this gives an equilibrium ionization density of $2 \times 10^{6} \mathrm{~cm}^{-3}$, which is clearly higher than the background ionization density in air, which ranges between $10^{2}$ and $10^{4} \mathrm{~cm}^{-3}$ depending on circumstances (e.g. radon concentration, presence of walls, cosmic radiation shielding).

At lower pressures, the ion-electron pair production rate decreases for two reasons: the absolute density of ${ }^{85} \mathrm{Kr}$ is lower, and the path length of the beta particles is longer. In other words, the ion-pair production rate scales with $n_{0}^{2}$ for gas densities $n_{0}$ when the penetration length is larger than the dimensions of the vessel. The equilibrium ionization densities will be roughly $4 \times 10^{5} \mathrm{~cm}^{-3}$ and $1 \times 10^{4} \mathrm{~cm}^{-3}$ at $200 \mathrm{mbar}$ and 25 mbar, respectively.

\subsection{General results}

We have compared discharges in the krypton-85-nitrogen mixture with discharges in pure nitrogen as discussed in [1]. The results are shown in figures 7-9. In general, the discharges are very similar to the discharges in pure nitrogen. However, there are some clear differences: in the krypton mixture, more branches occur. These 'extra' branches are longer than the protrusions observed in pure nitrogen and pure argon, but they 

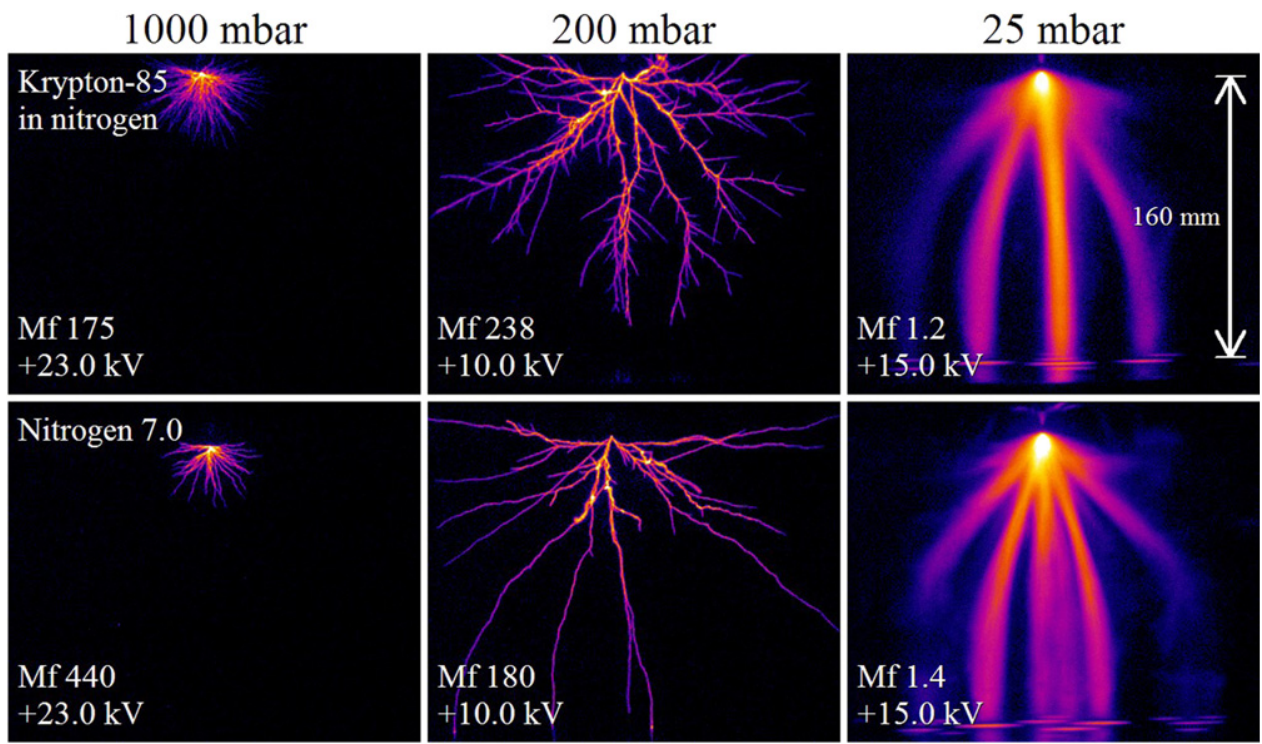

Figure 7. Comparison of streamer discharges in nitrogen with an admixture of ${ }^{85} \mathrm{Kr}$ and in nitrogen 7.0 at $1 \mathrm{~Hz}$ repetition rate. The discharges are produced with the C-supply. Discharges in other gas mixtures under the same conditions can be found in figure 5 from [1].

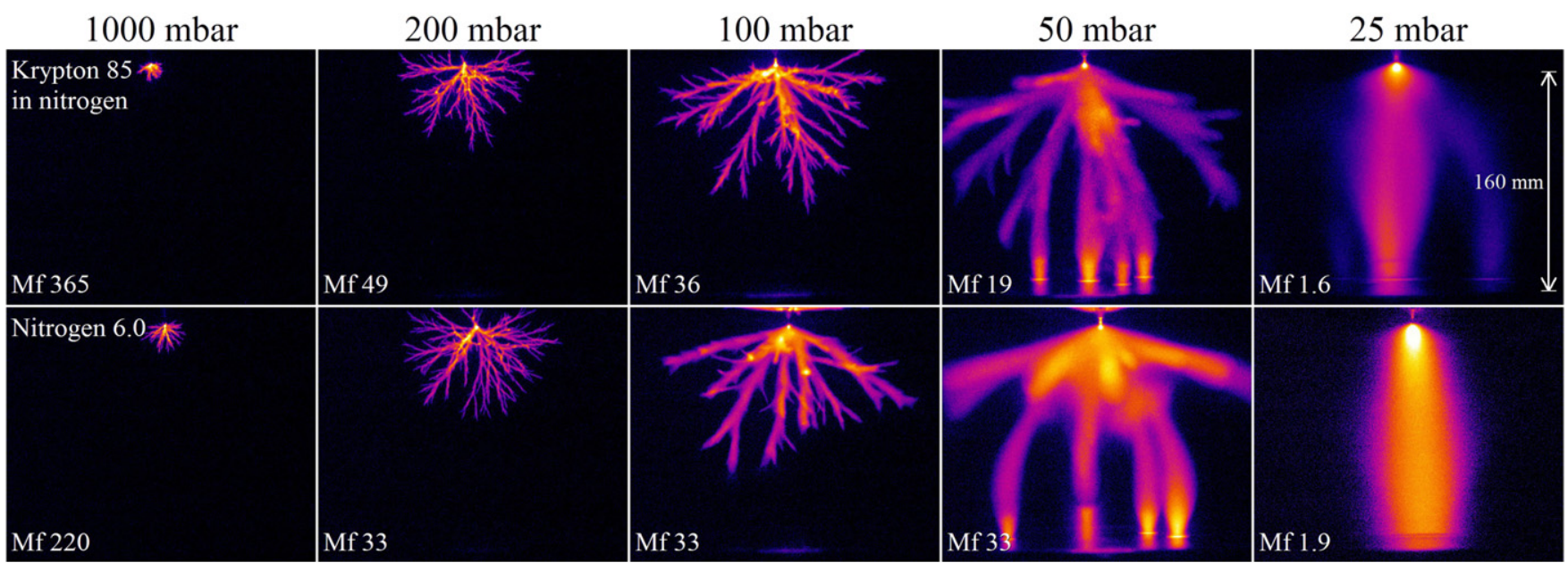

Figure 8. Comparison of streamer discharges in nitrogen with ${ }^{85} \mathrm{Kr}$ added and in pure nitrogen. The discharges are produced with the Blumlein pulser at about $20 \mathrm{kV}$ and $1 \mathrm{~Hz}$ repetition rate.

do not reach much farther than about 10 streamer diameters. This is especially clear in the 200 mbar images from figure 7 and the 50 and 100 mbar images from figure 8. The zoomed images at 200 mbar from figure 9 also show longer branches in the krypton mixture than the short protrusions in pure nitrogen. In the same figure, at 1000 mbar, the number of channels is about two times higher in the krypton- 85 mixture than in pure nitrogen, which may be attributed to the same reason.

However, the differences in general morphology between the krypton mixture and pure nitrogen have little influence on propagation velocities and minimal streamer diameters. As is shown in figure 10 , the measured propagation velocities and minimal streamer diameters in this mixture are very similar to values in other gas mixtures (see [1]). In this case, velocities are measured at the centre of the gap and diameters are determined from minimal streamers at relatively low voltages.

\subsection{Effects of repetition rate with ${ }^{85} \mathrm{Kr}$}

With the krypton-85-nitrogen mixture, we have performed similar experiments as discussed in section 3 . The results of these experiments are given in figure 11. Inception is very easy in this gas mixture. In all cases a full discharge was observed on the first pulse, also with lower voltage pulses (not shown here). This is no surprise, as the radioactive decay of the krypton-85 keeps supplying free electrons.

In figure 11, the morphology of discharges is very similar for $0.01,0.1$ and $1 \mathrm{~Hz}$ pulse repetition rates. The high density of protrusions that is observed at 0.1 and $0.01 \mathrm{~Hz}$ in pure nitrogen (see figure 3 ) is not visible in the krypton mixture. However, the thicker branches and less protrusions at $10 \mathrm{~Hz}$ that are observed in pure nitrogen are also visible in the krypton mixture at this repetition rate. A more quantitative approach to this is given in figure 12. Here, the number of protrusions (hairs or branches) per millimetre of streamer channel is plotted for 
the two gases at four repetition rates. Even though the method is quite crude (it is difficult to judge whether a structure is a feather and there has been no correction for the projection of the image), it gives the same general impression as the images themselves: streamers in pure nitrogen are more feather-like than in the nitrogen-krypton mixture, except at $10 \mathrm{~Hz}$ where both contain very few protrusions.

We can conclude from this that at $10 \mathrm{~Hz}$ repetition rate, the background ionization by previous discharges dominates over the background ionization by radioactivity and that therefore pure nitrogen cannot be distinguished from nitrogen with a radioactive admixture. If we use equation (11) to calculate the leftover ionization density at 200 mbar $1 \mathrm{~s}$ after a discharge, we get $2 \times 10^{6} \mathrm{~cm}^{-3}$. This is about five times higher than our estimation of background ionization caused by the krypton- 85 in our gas mixture, which is $4 \times 10^{5} \mathrm{~cm}^{-3}$ at 200 mbar. At
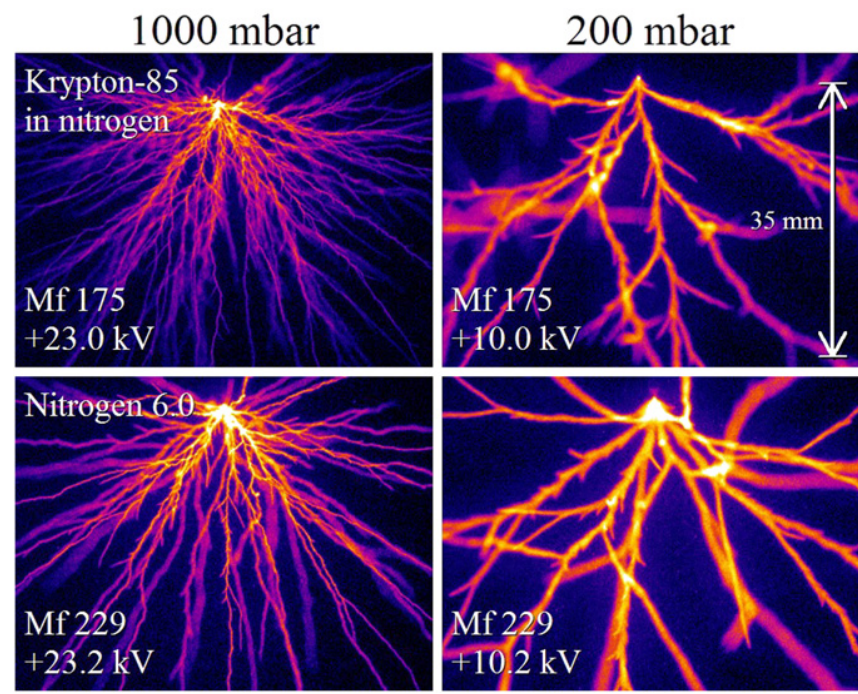

Figure 9. Comparison of zoomed streamer discharge images in nitrogen with ${ }^{85} \mathrm{Kr}$ added and in pure nitrogen, both at 1000 and 200 mbar around the anode tip region (see length indication at top right image). The discharges are produced with the C-supply at $1 \mathrm{~Hz}$ repetition rate.

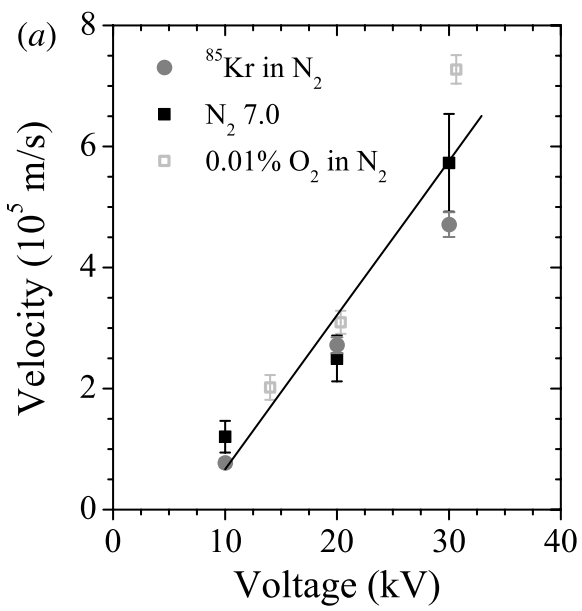

$10 \mathrm{~Hz}$ repetition rate however, the ionization left by previous discharges may dominate as the radioactive ionization remains constant, while the leftover ionization increases by a factor of 10 (compared with $1 \mathrm{~Hz}$ ). At repetition rates below $0.2 \mathrm{~Hz}$, the ionization density is dominated by krypton- 85 . Therefore, it is no surprise that we can no longer distinguish discharges at $1 \mathrm{~Hz}$ repetition rate from discharges at lower repetition rates, but that we still do see differences between discharges at 1 and $10 \mathrm{~Hz}$ repetition rate.

This confirms our estimates of the effects of repetition rate and radioactivity on background ionization levels. Together this makes for a consistent story.

However, all ionization densities are rough estimates and can have an uncertainty of up to one order of magnitude. Therefore, the repetition rate at which radioactive ionization dominates over ionization from discharge repetition has a similar uncertainty.

\subsection{Discussion}

The extra long branches observed in nitrogen with the radioactive admixture could be due to multiple ionization events in the trace of the fast electrons (beta particles) from the decay of krypton-85 (see section 5.1 for decay rates). However, in this case one would expect that their orientation is fully isotropic. We do not observe this isotropic behaviour. The extra branches are always orientated in a small angle (less than $45^{\circ}$ ) to the propagation direction of the streamer channel they are connected to. Still we cannot fully exclude this explanation as it may be possible that only traces in the direction of the field can form avalanches.

When we image a discharge in the krypton mixture with a short exposure time, as in figure 13, we never see any 'disconnected' parts of a streamer channel. If the long side channels in the krypton mixture would be avalanches moving towards the streamer head, one would expect to see small starting channels visually separated from the main channels. This does not occur in our images; we see short and long channels that are always connected to another channel. This

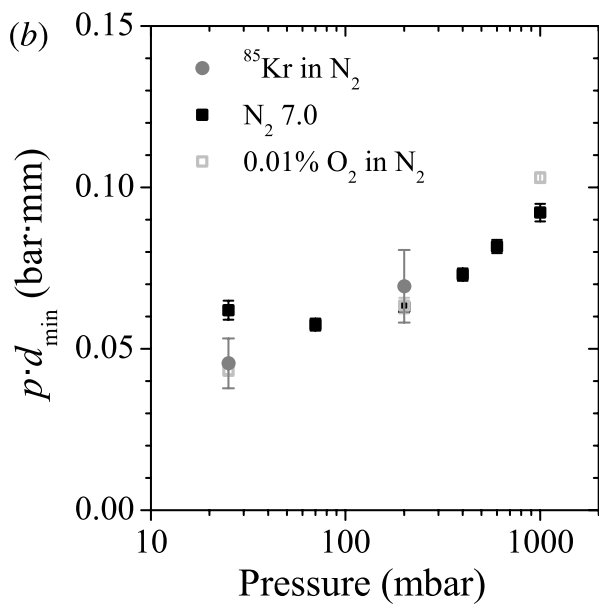

Figure 10. Streamers created with the C-supply at $1 \mathrm{~Hz}$ repetition rate: (a) velocities as a function of voltage at $200 \mathrm{mbar}$ and $(b)$ minimal streamer diameters as a function of pressure for the nitrogen-krypton mixture as well as for two other gases. The error bars indicate the sample standard deviation. The line in $(a)$ is a linear fit through all the points from figure 13 of [1]. More diameter measurements in nitrogen-oxygen mixtures are also given in [1]. 

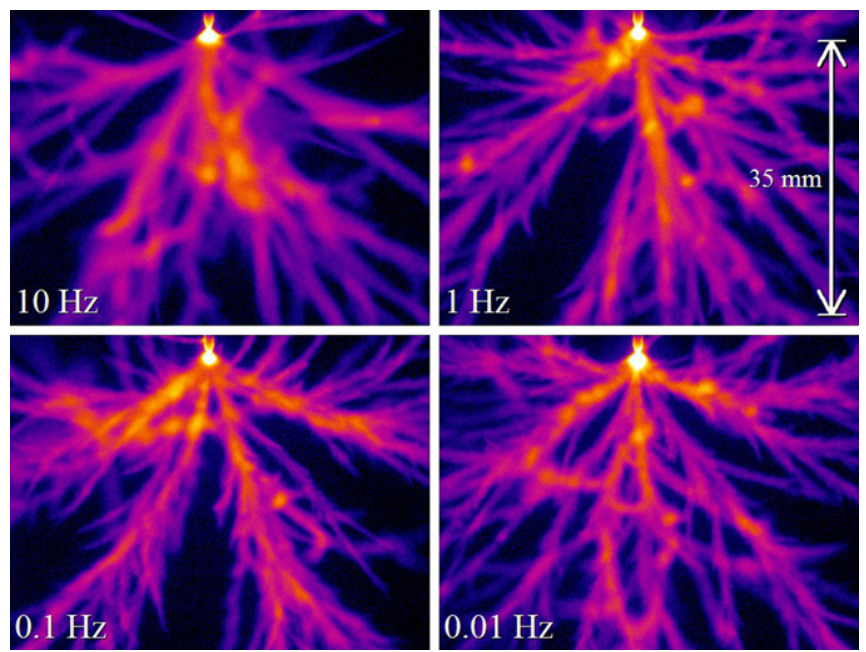

Figure 11. Zoomed images of the effects of pulse repetition rate on streamer morphology in the nitrogen-krypton- 85 gas mixture. These images are acquired at 200 mbar with the Blumlein pulser at $25 \mathrm{kV}$.

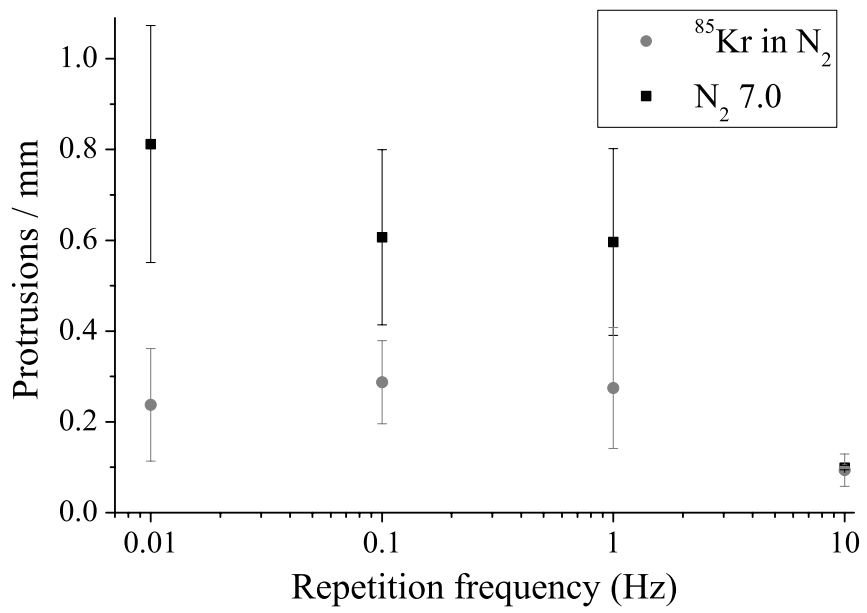

Figure 12. Number of protrusions per millimetre as a function of repetition rate for nitrogen 7.0 and the nitrogen-krypton mixture, both at 200 mbar. The same settings as in figures 3 and 11 are used. All visible irregularities and branches on a streamer channel are counted as protrusions (no corrections for projection or masking by the channel are made). The same zoomed images as those from figures 3 and 11 are used, with streamer channels at distances between 10 and $35 \mathrm{~mm}$ from the tip. For every point in the graph 2-4 images are used with 2-4 analysed streamer channels per image. The error bars indicate the standard deviation.

would indicate that the longer side channels in the krypton mixture are not avalanches themselves.

Clearly, an explanation of the observed structures requires a future investigation of the single particle dynamics of beta particles and ionization avalanches; it cannot be handled on the level of densities. Steps in this direction are taken in $[15,16,19]$.

\section{Conclusions}

We have found that the background ionization levels can have a significant influence on streamer inception, morphology and propagation. When background ionization levels are
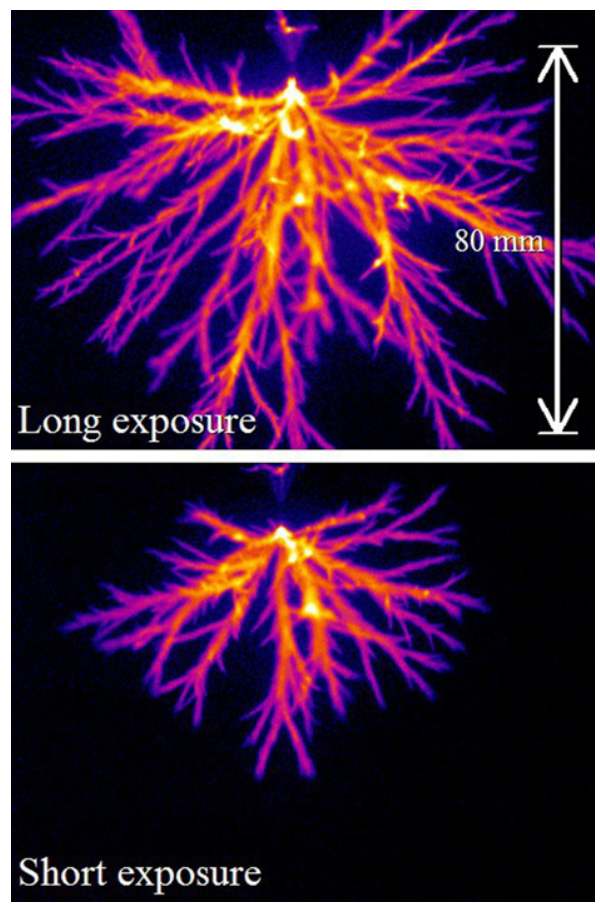

Figure 13. Overview images of streamer discharges in the nitrogen-krypton- 85 gas mixture at 200 mbar made with the Blumlein pulser at $25 \mathrm{kV}$. The top image is made with a long exposure so that the whole discharge evolution is visible. For the bottom image, the camera exposure duration was reduced to roughly $65 \mathrm{~ns}$ (half of the pulse duration).

lower due to lower pulse repetition rates, streamers in pure nitrogen branch more often and are more 'feather-like' in appearance. A similar effect was seen by Takahashi et al [5] and Mathew et al [6] when they varied background ionization locally by UV or x-ray radiation. The higher irregularity at lower background ionization levels is probably due to the more stochastic nature of the discharge. Lower ionization densities will lead to a lower avalanche density ahead of the streamer which more easily destabilizes the streamer front and therefore creates branching or feathers. Indeed, at the lowest ionization levels, the estimated number of avalanches created by single background electrons approximates the number of protrusions observed in very clean nitrogen $[1,2,14]$.

However, if all protrusions seen in the experiments were avalanches, one would expect the number of protrusions per length to increase with increasing background density, but figure 12 shows that the opposite is true. Therefore, the protrusions seen at higher background densities are probably not pure avalanches running towards the streamer, but they also could carry own space charge, and possibly they have to be identified with branches that extinguish after a short propagation length - future theoretical research and a closer inspection of the shape, length, brightness and evolution of the protrusions need to clarify this question. In artificial air we observe a puzzling effect as well: the inception cloud breaks up faster at higher repetition rates, i.e. at higher background ionization levels, while one would expect a stabilizing effect.

Could this be because at higher repetition rates the previous discharge has left an ionization trail behind that 
the next discharge will follow? Probably not, we have not observed any tendency of streamer channels to follow the same path as streamers in preceding voltage pulses. This was tested, in particular, in section 4 on streamers in 200 mbar nitrogen with $10 \mathrm{~Hz}$ repetition rate. We found that streamers at the next pulse search new paths. This agrees with our theoretical estimates in section 4.2 that even at the outer edges of the discharge, the ionization density in the streamer trail is only about three times above average at pulse repetition rates of $10 \mathrm{~Hz}$. (It should be noted, though, that there will be a substantial localized ionization trail at higher repetition rates.)

Background ionization left by previous discharges also influences the inception probability. We have not investigated this in depth, but our limited measurements show that in pure nitrogen at $0.01 \mathrm{~Hz}$ repetition rate (estimated leftover ionization density $n \approx 2 \times 10^{4} \mathrm{~cm}^{-3}$ ), the inception probability at voltage pulses of $25 \mathrm{kV}$ and $130 \mathrm{~ns}$ duration is in the order of $10 \%$, while at $0.1 \mathrm{~Hz}\left(n \approx 2 \times 10^{5} \mathrm{~cm}^{-3}\right)$ it is close to $100 \%$.

Theoretically, adding $9.9 \mathrm{ppb}$ of ${ }^{85} \mathrm{Kr}$ to pure nitrogen at 200 mbar in our vessel should create a background ionization level of about $4 \times 10^{5} \mathrm{~cm}^{-3}$. This value lies close to the background ionization density of $10^{6} \mathrm{~cm}^{-3}$ that was calculated in section 4.2 for a pulse repetition rate of $1 \mathrm{~Hz}$. Therefore, we expect to observe the effect of pulse repetition on streamer morphology in the ${ }^{85} \mathrm{Kr}$ mixture only at repetition rates of $1 \mathrm{~Hz}$ or higher. This is indeed what the experiments show. Therefore, our rough estimates of the background ionization levels due to pulse repetition or due to the radioactive admixture are consistent with each other.

In the ${ }^{85} \mathrm{Kr}$ mixture more short streamer sections appear. Whether the shape of the ionization traces from radioactive decay can explain this, has to be investigated in future work.

In general, our findings are in agreement with estimates and modelling by Pancheshnyi [3], Bourdon et al [12] and Wormeester et al [2]. They all find that background ionization has an influence on the structure and inception of streamers. Our experiments with adding radioactive ${ }^{85} \mathrm{Kr}$ to pure nitrogen and the comparison with streamer discharges at different repetition rates have confirmed that background ionization levels indeed play an important role in streamer propagation, but that their influence on propagation velocity and minimal streamer diameter is very limited.

\section{Acknowledgments}

GW acknowledges support by STW-project 10118, part of the Netherlands' Organization for Scientific Research NWO.

\section{References}

[1] Nijdam S, van de Wetering F M J H, Blanc R, van Veldhuizen E M and Ebert U 2010 Probing photo-ionization: experiments on positive streamers in pure gases and mixtures J. Phys. D: Appl. Phys. 43145204

[2] Wormeester G, Pancheshnyi S, Luque A, Nijdam S and Ebert U 2010 Probing photo-ionization: simulations of positive streamers in varying $\mathrm{N}_{2}: \mathrm{O}_{2}$-mixtures $J$. Phys. D: Appl. Phys. $\mathbf{4 3} 505201$
[3] Pancheshnyi S 2005 Role of electronegative gas admixtures in streamer start, propagation and branching phenomena Plasma Sources Sci. Technol. 14645

[4] Hartmann G and Gallimberti I 1975 The influence of metastable molecules on the streamer progression J. Phys. D: Appl. Phys. 8670

[5] Takahashi E, Kato S, Sasaki A, Kishimoto Y and Furutani H 2011 Controlling branching in streamer discharge by laser background ionization J. Phys. D: Appl. Phys. 44075204

[6] Mathew D, Bastiaens H M J, Boller K J and Peters P J M 2007 Effect of preionization, fluorine concentration, and current density on the discharge uniformity in $\mathrm{F}_{2}$ excimer laser gas mixtures J. Appl. Phys. 102033305

[7] Dyakonov M I and Kachorovskii V Y 1989 Streamer discharge in a homogeneous field Sov. Phys.-JETP 68 1070-4

[8] Raizer Yu P and Simakov A N 1998 Main factors determining the radius of the head of a long streamer and the maximum electric field near the head Plasma Phys. Rep. 24 700-6

[9] Dhali S K and Williams P F 1987 Two-dimensional studies of streamers in gases J. Appl. Phys. 62 4696-707

[10] Vitello P A, Penetrante B M and Bardsley J N 1994 Simulation of negative-streamer dynamics in nitrogen Phys. Rev. E 49 5574-98

[11] Luque A and Ebert U 2011 Density models for streamer discharges: beyond cylindrical symmetry and homogeneous media J. Comput. Phys. doi:10.1016/j.jcp.2011.04.019

[12] Bourdon A, Bonaventura Z and Celestin S 2010 Influence of the pre-ionization background and simulation of the optical emission of a streamer discharge in preheated air at atmospheric pressure between two point electrodes Plasma Sources Sci. Technol 19034012

[13] Luque A, Ratushnaya V and Ebert U 2008 Positive and negative streamers in ambient air: modeling evolution and velocities J. Phys. D: Appl. Phys. 41234005

[14] Wormeester G, Nijdam S and Ebert U 2011 Feather-like structures in positive streamers interpreted as electron avalanches Japan. J. Appl. Phys. 50 08JA01

[15] Li C, Ebert U and Hundsdorfer W 2009 3D hybrid computations for streamer discharges and production of run-away electrons J. Phys. D: Appl. Phys. 42202003

[16] Li C, Ebert U and Hundsdorfer W 2011 Spatially hybrid computations for streamer discharges: II. fully $3 \mathrm{D}$ simulations J. Comput. Phys. doi:10.1016/j.jcp.2011.07.023

[17] Li C, Ebert U and Hundsdorfer W 2011 Simulated avalanche formation around streamers in an overvolted air gap IEEE Trans. Plasma Sci. doi:10.1109/TPS.2011.2163528

[18] Chanrion O and Neubert T 2010 Production of runaway electrons by negative streamer discharges J. Geophys. Res. 115 A00E32

[19] Luque A and Ebert U 2011 Electron density fluctuations accelerate the branching of streamer discharges in air Phys. Rev. E at press arXiv:1102.1287

[20] Kossyi I A, Kostinsky A Y, Matveyev A A and Silakov V P 1992 Kinetic scheme of the non-equilibrium discharge in nitrogen-oxygen mixtures Plasma Sources Sci. Technol. 1207

[21] Hagelaar G J M and Pitchford L C 2005 Solving the boltzmann equation to obtain electron transport coefficients and rate coefficients for fluid models Plasma Sources Sci. Technol. 14722

[22] Dujko S, Ebert U, White R D and Petrović Z L 2011 Boltzmann equation analysis of electron transport in a $\mathrm{N}_{2}-\mathrm{O}_{2}$ streamer discharge Japan. J. Appl. Phys. 50 08JC01

[23] Aleksandrov N L and Bazelyan E M 1999 Ionization processes in spark discharge plasmas Plasma Sources Sci. Technol. 8285 
[24] Sentman D D, Stenbaek-Nielsen H C, McHarg M G and Morrill J S 2008 Plasma chemistry of sprite streamers J. Geophys. Res.-Atm. $113 \mathrm{D} 11112$

[25] Raizer Yu P 1991 Gas Discharge Physics (Berlin: Springer)

[26] Snuggs R M, Volz D J, Schummers J H, Martin D W and McDaniel E W 1971 Mobilities and longitudinal diffusion coefficients of mass-identified potassium ions and positive and negative oxygen ions in oxygen Phys. Rev. A 3 477-87

[27] Briels T M P, Kos J, van Veldhuizen E M and Ebert U 2006 Circuit dependence of the diameter of pulsed positive streamers in air J. Phys. D: Appl. Phys. 395201

[28] Ono R and Oda T 2003 Formation and structure of primary and secondary streamers in positive pulsed corona discharge-effect of oxygen concentration and applied voltage J. Phys. D: Appl. Phys. 36 1952-8

[29] Dubrovin D, Nijdam S, van Veldhuizen E M, Ebert U, Yair Y and Price C 2010 Sprite discharges on Venus and Jupiter-like planets: a laboratory investigation J. Geophys. Res.-Space Phys. 115 A00E34

[30] Goldman M and Goldman A 1978 Corona discharges Gaseous Electronics vol 1 Electrical Discharges ed M N Hirsh and H J Oskam (New York: Academic) p 219

[31] van Veldhuizen E M and Rutgers W R 2003 Inception behaviour of pulsed positive corona in several gases $J$. Phys. D: Appl. Phys. 362692

[32] Hermans P J 2004 Een studie naar het gedrag van corona-ontladingen in verschillende gassen Trainee Report Eindhoven University of Technology EPG 04-01

[33] Liu Z, van Veldhuizen E M, van Heesch E J M and Pemen A J M 2011 Strong density gradients in postdischarges in argon and air IEEE Trans. Plasma Sci. doi:10.1109/TPS.2011.2156418
[34] Starikovskii A Y, Anikin N B, Kosarev I N, Mintoussov E I, Nudnova M M, Rakitin A E, Roupassov D V, Starikovskaia S M and Zhukov V P 2008 Nanosecond-pulsed discharges for plasma-assisted combustion and aerodynamics J. Propul. Power 241182

[35] Ratushnaya V, Luque A and Ebert U 2011 Electrodynamic characterization of long positive streamers in air J. Phys. D: Appl. Phys. submitted

[36] Nikipelov A, Popov I B, Pancheshnyi S and Starikovskii A Yu 2011 Localized and distributed behavior of nanosecond pulse-periodic discharge in air Proc. 30th ICPIG (Belfast, UK) $\mathrm{pp} \mathrm{C} 10-346$

[37] Celestin S, Bonaventura Z, Zeghondy B, Bourdon A and Ségur P 2009 The use of the ghost fluid method for poisson's equation to simulate streamer propagation in point-to-plane and point-to-point geometries J. Phys. D: Appl. Phys. 42065203

[38] Briels T M P, van Veldhuizen E M and Ebert U 2008 Positive streamers in air and nitrogen of varying density: experiments on similarity laws J. Phys. D: Appl. Phys. 41234008

[39] Fries B A 1977 Krypton-85. a versatile tracer for industrial process applications Int. J. Appl. Radiat. Isot. 28 829-32

[40] Gallagher T, Silva J, Almeida T, Baselice M, Yang M and Chaudhari P 2010 Krypton-85 gas mixture radioactivity comparisons Proc. 12th Int. Symp. on the Science and Technology of Light Sources (Eindhoven, The Netherlands, 2010)

[41] Glendenin L E 1948 Determination of the energy of beta particles and photons by absorption Nucleonics $\mathbf{2} 12$

[42] L'Annunziata M F 2003 Handbook of Radioactivity Analysis (San Diego: Academic) 